\title{
Active galactic nuclei in the mid-IR
}

\section{Evolution and contribution to the cosmic infrared background ${ }^{\star}$}

\author{
I. Matute ${ }^{1,2}$, F. La Franca ${ }^{2}$, F. Pozzi ${ }^{3,4}$, C. Gruppioni ${ }^{4}$, C. Lari ${ }^{5}$, and G. Zamorani ${ }^{4}$
}

\author{
1 Max-Planck Institut für extraterrestrische Physik (MPE), Giessenbachstraße, Postfach 1312, 85741 Garching, Germany \\ e-mail: matute@mpe.mpg.de \\ 2 Dipartimento di Fisica, Università degli Studi "Roma Tre", via della Vasca Navale 84, 00146 Roma, Italy \\ e-mail: lafranca@fis.uniroma3.it \\ 3 Dipartimento di Astronomia, Università di Bologna, via Ranzani 1, 40127 Bologna, Italy \\ 4 INAF, Osservatorio Astronomico di Bologna, via Ranzani 1, 40127 Bologna, Italy \\ 5 INAF, Istituto di Radioastronomia (IRA), via Gobetti 101, 40129 Bologna, Italy
}

Received 28 June 2005 / Accepted 30 December 2005

\section{ABSTRACT}

Aims. We study the evolution of the luminosity function (LF) of type-1 and type-2 Active Galactic Nuclei (AGN) in the mid-infrared, derive the contribution of the AGN to the Cosmic InfraRed Background (CIRB) and the expected source counts to be observed by Spitzer at $24 \mu \mathrm{m}$. Methods. We used a sample of type-1 and type-2 AGN selected at $15 \mu \mathrm{m}$ (ISO) and $12 \mu \mathrm{m}$ (IRAS), and classified on the basis of their optical spectra. Local spectral templates of type- 1 and type- 2 AGN have been used to derive the intrinsic $15 \mu$ m luminosities. We adopted an evolving smooth two-power law shape of the LF, whose parameters have been derived using an un-binned maximum likelihood method.

Results. We find that the LF of type-1 AGN is compatible with a pure luminosity evolution $\left(L(z)=L(0)(1+z)^{k_{L}}\right)$ model where $k_{L} \sim 2.9$. A small flattening of the faint $\left(L_{15}<L_{15}^{*}\right)$ slope of the LF with increasing redshift is favoured by the data. A similar evolutionary scenario is found for the type-2 population with a rate $k_{L}$ ranging from $\sim 1.8$ to 2.6, depending significantly on the adopted mid-infrared spectral energy distribution. For type-2 AGN a flattening of the LF with increasing redshift is suggested by the data, possibly caused by the loss of a fraction of type- 2 AGN hidden within the optically classified starburst and normal galaxies. The type- 1 AGN contribution to the CIRB at $15 \mu \mathrm{m}$ is (4.2-12.1) $\times 10^{-11} \mathrm{~W} \mathrm{~m}^{-2} \mathrm{sr}^{-1}$, while the type-2 AGN contribution is $(5.5-11.0) \times 10^{-11} \mathrm{~W} \mathrm{~m}^{-2} \mathrm{sr}^{-1}$. We expect that Spitzer will observe, down to a flux limit of $S_{24 \mu \mathrm{m}}=0.01 \mathrm{mJy}$, a density of $\sim 1200 \mathrm{deg}^{-2}$ type-1 and $\sim 1000 \mathrm{deg}^{-2}$ type-2 optically classified AGN.

Conclusions. AGN evolve in the mid-infrared with a rate similar to the ones found in the optical and X-rays bands. The derived total contribution of the AGN to the CIRB (4-10\%) and Spitzer counts should be considered as lower limits, because of a possible loss of type-2 sources caused by the optical classification.

Key words. cosmology: observations - infrared: galaxies - galaxies: active - surveys - galaxies: evolution

\section{Introduction}

Active Galactic Nucleus (AGN hereafter) are expected to have played an important role in the formation and evolution of the galaxies in the Universe. An example is the observation of the correlation between the mass of the central black holes $\left(M_{\mathrm{BH}}\right)$ and the mass of the bulges (Magorrian et al. 1998) or the velocity dispersion of gas and stars in the bulges of galaxies $\left(M_{\mathrm{BH}}-\sigma\right.$ relation; Ferrarese \& Merritt 2000; Tremaine et al. 2002). Thus, the measure of the history of the density of AGN as a function of the luminosity (the luminosity function, LF hereafter) can provide fundamental clues to explain the present day universe

^ Data presented here are based on observations by the Infrared Space Observatory (ISO). ISO is an ESA project with instruments funded by ESA member states (especially the PI countries: France, Germany, the Netherlands and the United Kingdom) and with the participation of ISAS and NASA. (e.g. Balland et al. 2003; Menci et al. 2004; Granato et al. 2004; Di Matteo et al. 2005).

The LF will not only provide information on the census of AGN, but will also place constraints on the physical model of AGN, the origin and accretion history into supermassive black holes and the formation of structures in the early universe. Moreover, the measure of the AGN LF over the whole spectral range will provide information on the relative importance of accretion power in the overall energy budget of the universe.

AGN have been historically classified into two groups (type-1 and type-2), depending on the presence or absence in their optical spectra of broad emission lines ( FWHM > $1200 \mathrm{~km} \mathrm{~s}^{-1}$ ). The unified model for AGN assumes that the two types of sources are intrinsically the same (Antonucci et al. 1993), and that the observed differences in the optical spectra are explained by an orientation effect. The presence 
of obscuring material (torus or warped disc) around the central energy source and its orientation with respect to the observer could prevent a direct view of the central region around the nuclei responsible for the broad line emission observed in the optical band. AGN are then classified in two groups: the un-obscured (type-1) and obscured (type-2) sources depending on whether the line-of-sight intersects the obscuring material or not. However, it seems clear that the orientation-dependent model is a 0th-order approximation to the true nature of AGN (e.g. Barger et al. 2005; La Franca et al. 2005).

The major efforts to understand the evolution of AGN historically have been concentrated in optical wavelengths and in recent years have produced the largest compilations of AGN from the Two Degree Field (2dF; Boyle et al. 2000; Croom et al. 2004) and the Sloan Digital Sky Survey (SDSS; York et al. 2000; Hao et al. 2005). However the optical bands have proved to be very inefficient in the selection of obscured sources and the assembly of unbiased samples of type- 2 objects is difficult. In recent years many new windows have been opened at various wavelength bands for the observation of high redshift galaxies and AGN: sub-mm (SCUBA), Infrared (IRAS, ISO and Spitzer) and the X-ray (ROSAT, XMM and Chandra). Thanks to their smaller dependence on dust obscuration compared to the optical surveys, the Infrared and the hard X-ray wavelengths have proved to be much more efficient in the detection of type- 2 sources.

X-ray observations so far have been consistent with population synthesis models based on the 0th-order unified AGN scheme (e.g. Comastri et al. 1995) or its modifications (Pompilio et al. 2000; Gilli et al. 2001; Ueda et al. 2003; La Franca et al. 2005). The hard spectrum of the X-ray background is explained by a mixture of absorbed and unabsorbed AGN, evolving with cosmic time. According to these models, most AGN spectra should be heavily obscured as the light produced by accretion is absorbed by gas and dust.

For this reason an important mid-infrared $(4-40 \mu \mathrm{m})$ thermal emission is expected from reprocessed radiation by gas and dust grains directly heated by the central black hole (e.g. Granato et al. 1997; Oliva et al. 1999; Nenkova et al. 2002). AGN in this case could be important contributors to the Cosmic Infrared Background (CIRB). Indeed, IRAS has observed strong mid-IR emission in all local $(z<0.1)$ AGN (Miley et al. 1985; de Grijp et al. 1985; Neugebauer et al. 1986; Sanders et al. 1989). Complete and largely unbiased samples of AGN have been produced at $12 \mu \mathrm{m}$ (Rush et al. 1993, RMS hereafter) and $25 \mu \mathrm{m}$ (Shupe et al. 1998) based on IRAS observations. These samples provide an important insight into the infrared emission from local galaxies and a firm basis with which to compare the properties of galaxies in the local universe with the high redshift populations uncovered by ISO $(z \leq 1)$ and most recently by Spitzer $(z \leq 2)$.

Statistical studies of AGN in the mid-IR have been based on large but local samples from the IRAS observations (e.g. RMS, Shupe et al. 1998) and deep but small ISO fields in the Hubble Deep Field North (HDFN; Aussel et al. 1999a,b), South (HDFS; Oliver et al. 2002) and the Canada-France Redshift Survey (CFRS; Flores et al. 1999) that provided only a handful of objects.
Therefore, due to the small number of high redshift objects available, the shape and evolution of the mid-IR LF of these objects at high redshift was largely unknown. Only recently, large and highly complete $15 \mu \mathrm{m}$ spectroscopic samples, based on the European Large Area ISO Survey (ELAIS hereafter; Oliver et al. 2000), have been released (Rowan-Robinson et al. 2004; La Franca et al. 2004, FLF04 hereafter). Based on the analysis of the ISO observations in the ELAIS-S1 field, Matute et al. (2002, M02 hereafter) derived the first estimate of the type-1 LF in the mid-IR $(15 \mu \mathrm{m})$, finding an evolution similar to the ones observed in the optical (Croom et al. 2004) and X-rays (e.g. Hasinger et al. 2005; La Franca et al. 2005). The evolution of the LF for normal and starburst galaxies detected at $15 \mu \mathrm{m}$ in the southern ELAIS fields (S1 and S2) has been derived and discussed by Pozzi et al. (2004).

In this paper we investigate the separate evolution of type-1 and type-2 AGN in the mid-IR and their contribution to the CIRB using: $a$ ) the local IR population uncovered by IRAS; $b$ ) deep observations from ISO fields and; $c$ ) the most recent spectroscopic identifications of $15 \mu \mathrm{m}$ sources in the southern fields of the ELAIS Survey (Pozzi et al. 2003; FLF04) ${ }^{1}$. The AGN sample used in our work is described in Sect. 2. Section 3 introduces the general method used to derive the parameters of the luminosity function and presents the results. In Sect. 4 we discuss our results and compare them with the LFs derived in the mid-IR by previous analysis of mid-IR observations. The contribution to the CIRB and the predicted counts in the mid-IR Spitzer band at $24 \mu \mathrm{m}$ are computed and discussed in Sects. 5 and 6 . Section 7 summarises the main results.

A cosmology with $H_{0}=75 \mathrm{~km} \mathrm{~s}^{-1} \mathrm{Mpc}^{-1}$ and $\Omega_{\lambda}=0.7$, $\Omega_{\mathrm{m}}=0.3$ was adopted for the work presented in this paper.

\section{The sample and source classification}

The mid-IR selected AGN sample used in this work has been extracted from: $(i)$ the $15 \mu \mathrm{m}$ ELAIS fields S1 and S2 (Lari et al. 2001; Pozzi et al. 2003), (ii) the $15 \mu \mathrm{m}$ deep ISO surveys in the HDFN (Aussel et al. 1999a,b), HDFS (Oliver et al. 2002) and CFRS (Flores et al. 1999), (iii) the local IRAS $12 \mu \mathrm{m}$ sample of RMS.

The $15 \mu \mathrm{m}$ catalogue in the ELAIS S1 field was released by Lari et al. (2001). It covers an area of $\sim 4 \mathrm{deg}^{2}$ centred at $\alpha(2000)=00^{\mathrm{h}} 34^{\mathrm{m}} 44.4^{\mathrm{s}}, \delta(2000)=-43^{\circ} 28^{\prime} 12^{\prime \prime}$ and includes 462 mid-IR sources down to a flux limit of $0.5 \mathrm{mJy}$. Mid-IR source counts based on this catalogue have been presented and discussed by Gruppioni et al. (2002).

The analysis presented here is restricted to the more reliable subsample of 406 sources as described by FLF04. About $80 \%$ of these sources have been optically identified on CCD exposures down to $R \sim 23$, while spectral classification has been obtained for $90 \%$ of the optically identified sample. As discussed by FLF04, due to a different mid-IR flux limit coverage of the ELAIS-S1 field, the total area has been divided into two regions: the central and deepest region of S1 (S1-5) reaching mid-IR fluxes ( $S_{15}$ hereafter) of $0.5 \mathrm{mJy}$, and the outer region

\footnotetext{
${ }^{1}$ Data and related papers about the ELAIS southern survey are available at: http://www.fis. uniroma3.it/ ELAIS_S
} 
(S1-rest) with a 0.9 mJy flux limit. The $\mathrm{S} 1-5$ area is spectroscopically complete at the $97 \%$ level down to $R=21.6$, while S1-rest completeness reaches the $98 \%$ level down to $R=20.5$. In total 116 sources ( $29 \%$ of the total mid-IR sample) do not have a spectroscopic identification due to incompleteness of the follow-up or to the lack of optical counterpart brighter than $R=23$. A detailed description of the optical identification, spectroscopic classification, size and completeness function of the different areas used in the ELAIS-S1 sample are presented and discussed by FLF04.

The S2 field is a smaller and deeper area centred at $\alpha(2000)=05^{\mathrm{h}} 02^{\mathrm{m}} 24.5^{\mathrm{s}}, \delta(2000)=-30^{\circ} 36^{\prime} 00^{\prime \prime}$ covering $\sim 0.12 \mathrm{deg}^{2}$. This region includes 43 sources with $S / N>5$ down to $S_{15}=0.4 \mathrm{mJy}$ (Pozzi et al. 2003; Rowan-Robinson et al. 2004). Photometry in the whole field is available in the $U, B, I$ and $K^{\prime}$ bands down to 21.0, 24.5, 22.0 and 18.75 respectively (Vega magnitudes). 39 infrared sources have a counterpart in the $I$ band. 22 of them have a spectroscopic identification, while 8 sources are classified as stars since they are associated with bright $(I<14)$ point-like sources in the optical catalogue. To avoid large incompleteness due to the optical follow-up, only sources with optical counterparts brighter than $I=20.6$ were selected. Down to this optical limit, the sample considered in $\mathrm{S} 2$ is then complete at the $95 \%$ spectroscopic level for sources with $S_{15}>0.7 \mathrm{mJy}$.

Classification for AGN dominated sources in the ELAIS fields was based on their optical spectral signatures. Sources showing broad emission line profiles (rest frame FWHM > $1200 \mathrm{~km} \mathrm{~s}^{-1}$ ) were classified as type-1. Type-2 sources were selected following classic diagnostic diagrams (e.g. Tresse et al. 1996; Osterbrock 1989; Veilleux \& Osterbrock 1987) that included one or more of the following line ratios: $[\mathrm{NII}] / \mathrm{H} \alpha, \mathrm{SII} /(\mathrm{H} \alpha+[\mathrm{NII}]), \mathrm{OI} / \mathrm{H} \alpha, \quad[\mathrm{OIII}] / \mathrm{H} \beta$ and $[\mathrm{OII}] / \mathrm{H} \beta$ when available, depending on the redshift of the source (e.g. $\log ([\mathrm{OIII}] / \mathrm{H} \beta)>0.5$ and $\log ([\mathrm{NII}] / \mathrm{H} \alpha)>-0.4)$.

In total, the southern ELAIS $(\mathrm{S} 1+\mathrm{S} 2)$ AGN sample includes $27(25+2)$ type- 1 and $25(23+2)$ type-2 AGN representing $\sim 16 \%(52 / 320)$ of the identified infrared sources and $\sim 24 \%$ $(52 / 221)$ of the extragalactic population. Their distribution in the redshift- $R$-magnitude space is shown in Fig. 1 . The plotted lines represent a linear fit $( \pm 1 \sigma)$ to the data (dotted for type-1 AGN and dashed for type-2). Type-1 sources are detected up to $z \sim 3$ and show a quasi redshift-independent and narrow optical magnitude distribution $(R=18.76 \pm 1.01)$. On the other hand, type- 2 sources show a larger spread of optical magnitudes $(R=18.45 \pm 1.62)$ and a rather steep dependence on the redshift up to $z \sim 1$.

Constraints on the fainter population are provided by deep $15 \mu \mathrm{m}$ observations in the HDFN (Aussel et al. 1999a,b), HDFS (Oliver et al. 2002) and in the $1452+52$ field of the CFRS (Flores et al. 1999). These fields have a flux limit about an order of magnitude deeper $\left(S_{15} \simeq 0.1 \mathrm{mJy}\right)$ than the ELAIS-S fields but with sky coverages 100 to 500 times smaller. The multiwavelength follow-up in these fields has identified a small fraction of sources as AGN (both type-1 and type-2).

In the HDFN, $4115 \mu \mathrm{m}$ sources have been detected over an area of $21.5 \mathrm{arcmin}^{2}$. The sample is complete down to 0.1 mJy (Aussel et al. 1999a). 4 of these sources, with

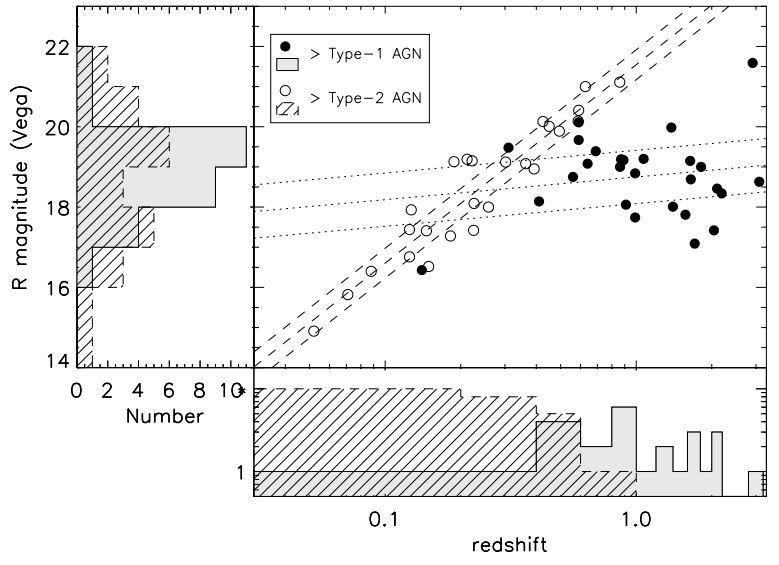

Fig. 1. Distribution of ELAIS-S1 type-1 and -2 AGN in the $z-R$ magnitude plane. Best fit linear solutions $( \pm 1 \sigma)$ in the $\log (z)-R$ space are shown as dotted lines for type-1 AGN and as dashed lines for type2 AGN. The $R$-magnitude and redshift distributions are shown in the left and bottom panels respectively.

fluxes between 0.1 and $0.45 \mathrm{mJy}$, are found to be AGN by Alexander et al. (2002), equally divided between the two classes (Alexander et al., private communication). In the HDFS we use the AGN identified by Franceschini et al. (2003) from a sample of 59 ISOCAM sources with $S_{15} \geq 0.1$ mJy selected over an area of $19.6 \mathrm{arcmin}^{2}$. They have detected two secure AGN, one type-1 $\left(S_{15}=0.288 \mathrm{mJy}\right)$ and one type-2 $\left(S_{15}=\right.$ $0.518 \mathrm{mJy}$ ). The observations in the CFRS $1452+52$ field cover an area of $\sim 100 \mathrm{arcmin}^{2} .41$ sources with $S_{15} \geq 0.35 \mathrm{mJy}$ and with $S / N \geq 4$ have been selected (Flores et al. 1999). The spectroscopic follow-up identified one type- $1\left(S_{15}=0.459 \mathrm{mJy}\right)$ and one type-2 $\left(S_{15}=1.653 \mathrm{mJy}\right)$ source. In the abovementioned fields, a fraction of the type- 2 sources were not classified according to their optical spectra but on the basis of the shape of their multiwavelength spectral energy distribution (SED). For this reason only the selected type-1 population has been used in our analysis.

We combined the ISOCAM data with the local IRAS sample of AGN from RMS. The RMS Catalogue is a high galactic latitude $\left(|b| \geq 25^{\circ}\right)$, colour selected $\left(S_{60 \mu \mathrm{m}} / S_{12 \mu \mathrm{m}}\right)$ and flux limited $\left(S_{12 \mu \mathrm{m}}>0.22 \mathrm{Jy}\right)$ sample extracted from the IRAS $12 \mu \mathrm{m}$ Faint Source Catalog, Version 2 (Moshir et al. 1991). To avoid completeness uncertainties in the mid-IR sample, only sources with $S_{12 \mu \mathrm{m}} \geq 300 \mathrm{mJy}$ were selected (see RMS). In total 41 type- 1 and 50 type-2 sources are found within this flux range. The photometric and spectroscopic follow-up of the optical counterparts is considered to be $\sim 100 \%$ complete for these sources and has been used in our analysis as representative of the mid-IR selected population of AGN in the local universe.

\section{The evolution of AGN}

\subsection{The method}

In order to compare local sources with a higher redshift selected population and to probe changes with cosmic time it is necessary to compare their intrinsic physical properties. Our sample has been selected in the mid-IR and identified in the optical band, therefore rest-frame luminosities, in the form of $v L_{v}$, 
at $15 \mu \mathrm{m}\left(L_{15}\right)$ and in the $R$-band $\left(L_{R}\right)$ were computed using well known SEDs for type-1 and 2 sources.

The compilation of Elvis et al. (1994) of 47 QSO, in the range of $1-20 \mu \mathrm{m}$, was used as representative of the mid-IR emission for the type-1 population. This composite SED agrees very well with spectroscopic observations of type-1 sources performed by ISO in the mid-IR (Clavel et al. 2000; Spoon et al. 2002), showing a strong power-like continuum, very weak or no PAH emission bands and no evidence of the $10 \mu \mathrm{m}$ silicate absorption feature. Optical luminosities for type-1 AGN were computed using the $R$-band $k$-correction by Natali et al. (1998), which takes into account the large effect of broad lines entering and leaving the passband.

Unlike type-1 sources, the mid-IR SED of type-2 objects vary greatly. They range from starburst-like galaxy SEDs, as in the case of Circinus (Sturm et al. 2000), showing a weak continuum, prominent emission from PAH molecules and a deep silicate absorption feature at $\sim 10 \mu \mathrm{m}$, to more power-like SEDs dominated by hot dust directly heated by the active nucleus, as in NGC 1068 (Sturm et al. 2000). As a consequence, the midIR SED of NGC 1068 and Circinus galaxies (Fig. 2) were used as representative of two extreme cases of obscured AGN in the mid-IR. In order to derive the optical $k$-correction for type- 2 sources, an average spectrum was produced in the optical band (3500-7000 ̊) using our best type-2 spectra (Fig. 5 in FLF04).

In Fig. 3 we show the distributions in the $L_{15}-z$ plane of the total sample used in our analysis, while Table 1 summarises the mean redshifts and mid-IR luminosities, and corresponding $1 \sigma$ dispersion, measured for the local (IRAS) and high redshift (ISO) sources.

The adopted shape of the luminosity function (LF) is a smooth two-power law of the form:

$$
\frac{\mathrm{d} \Phi\left(L_{15}, z\right)}{\mathrm{d} \log L_{15}}=\frac{\Phi^{*}}{\left(L_{15} / L_{15}^{*}\right)^{\alpha(z)}+\left(L_{15} / L_{15}^{*}\right)^{\beta}}
$$

where we have introduced a dependence on $z$ in order to allow a change in the slope $(\alpha)$ of the faint end of the luminosity function. We have assumed a luminosity evolution of the form:

$L_{15}(z)= \begin{cases}L_{15}(0)(1+z)^{k_{L}} & \text { for } z \leq z_{\text {cut }} \\ L_{15}\left(z_{\text {cut }}\right) & \text { for } z>z_{\text {cut }}\end{cases}$

where $z_{\text {cut }}$ is the redshift cut beyond which the luminosity function is assumed to remain constant.

The parameters for the luminosity function and the evolution have been derived using an un-binned, maximum likelihood method (Marshall et al. 1983), as described by Matute et al. (2002). The spectro-photometric follow-up of the ELAIS southern fields was not deep enough to provide an identification and a classification for all the sources detected in the mid-IR sample. A factor depending on redshift and mid-IR luminosity was therefore introduced in order to take into account the fraction of objects not identified, due to the lack of an optical counterpart or to incompleteness of the spectroscopic follow-up. We will refer to this factor as the optical-completeness factor, $\boldsymbol{\Theta}(z, L)$. It represents the probability that a source with a given redshift and mid-IR luminosity $L_{15}$ has an apparent $R$-band magnitude within the spectroscopic limits of the samples, and
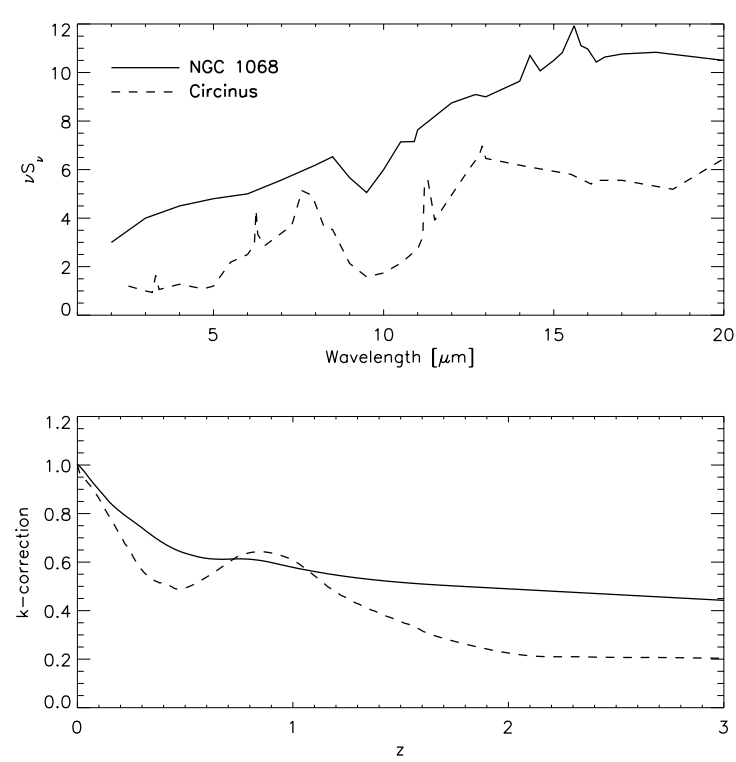

Fig. 2. Adopted mid-IR spectral energy distributions (SEDs) for type2 sources (top panel) and the corresponding $k$-corrections as a function of redshift computed for the ISOCAM-LW3 filter (bottom panel).

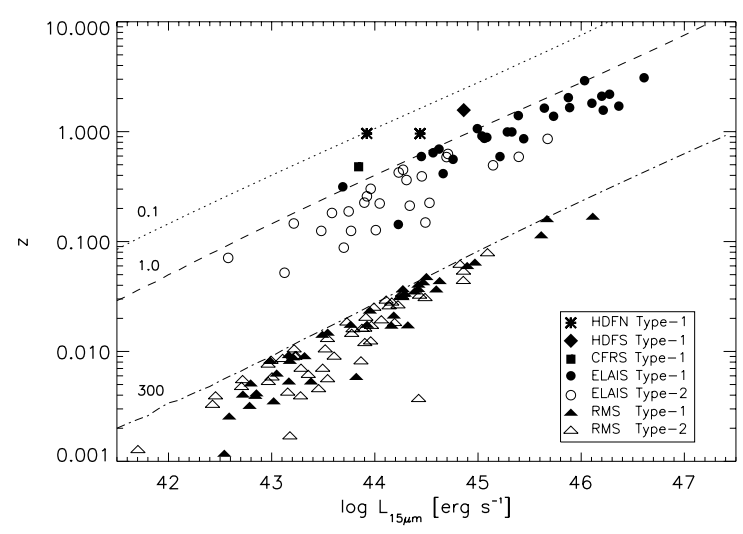

Fig. 3. Distribution in the $L_{15}-z$ space of the total sample of mid-IR type-1 and 2 AGN. The mid-IR luminosity for type- 2 sources has been computed applying the Circinus $k$-correction (see text, Sect. 3.1). The dotted, dashed and dot-dashed lines represent the flux limits at 0.1, 1.0 and $300 \mathrm{mJy}$ respectively.

was derived taking into account both the average $L_{15} / L_{R}$ ratio of the sources and its observed natural spread. For any given midIR luminosity $L_{15}$, this probability has been computed assuming a Gaussian distribution of $\log \left(L_{15} / L_{R}\right)$ centred at its mean value and with a sigma equal to the observed natural spread of the $L_{15} / L_{R}$ relation (see next sections for discussions on the adopted mean values and spreads of $\left.\log \left(L_{15} / L_{R}\right)\right)^{2}$. Then, the function to be minimised can be written as:

$S=-2 \sum_{i=1}^{\mathrm{N}} \ln \left[\Phi\left(z_{i}, L_{i}\right)\right]+2 \iint \Phi(z, L) \Omega(z, L) \Theta(z, L) \frac{\mathrm{d} V}{\mathrm{~d} z} \mathrm{~d} z \mathrm{~d} L$

where $\Omega(z, L)$ is the effective area covered by each subsample at $15 \mu \mathrm{m}, L=L_{15}$ and the index $i$ runs over all the objects of a given class. For the ELAIS-S1 sources the area coverage is

2 A similar approach was adopted in the previous estimates of the mid-IR type-1 AGN LF by Matute et al. (2002), and of the X-ray AGN LF by La Franca et al. (2002, 2005). 
Table 1. Mean redshift and luminosity $( \pm 1 \sigma)$ of the mid-IR samples.

\begin{tabular}{ccccc}
\hline \hline Sample & \multicolumn{2}{c}{ IRAS } & \multicolumn{2}{c}{ ISO } \\
& $\langle z\rangle$ & $\left\langle\log L_{15}\right\rangle^{a}$ & $\langle z\rangle$ & $\left\langle\log L_{15}\right\rangle$ \\
\hline Type-1 & $0.03 \pm 0.02$ & $43.87 \pm 0.87$ & $1.23 \pm 0.71$ & $45.22 \pm 0.78$ \\
Type-2 & $0.02 \pm 0.01$ & $43.66 \pm 0.68$ & $0.28 \pm 0.20$ & $43.90 \pm 0.69$ \\
\hline
\end{tabular}

a $12 \mu \mathrm{m}$ luminosities from rms have been converted to $15 \mu \mathrm{m}$ using the adopted SED. In particular the Circinus SED was adopted for type-2 AGN (see Sect. 3.1).

presented as a function of flux by FLF04. Area coverages for the deeper ISO fields (HDF-N, -S and CFRS) are described in the previous section. The area sampled by RMS is considered equal to 0 for $S_{12}<300 \mathrm{mJy}$ and equal to $22191.80 \mathrm{deg}^{2}$ for $S_{12} \geq 300 \mathrm{mJy}$ (see RMS for details). The representative SEDs were used to convert the rms $12 \mu \mathrm{m}$ fluxes into $15 \mu \mathrm{m}$ fluxes.

Confidence regions for each parameter have been obtained by computing $\Delta S\left(=\Delta \chi^{2}\right)$ at a number of values around the best parameter $\left(S_{\min }\right)$, while allowing the other parameters to float (see Lampton et al. 1976). A $68 \%$ confidence region for any single parameter corresponds to $\Delta S=1$. The goodness of the fit has been verified using the bidimensional Kolmogorov-Smirnov test (2D-KS hereafter) in the $L_{15}-z$ space (see Peacock 1983; Fasano \& Franceschini 1987). The normalisation factor $\Phi^{*}$ is determined in such a way to reproduce the observed total number of sources (ISO + IRAS).

\subsection{The evolution of type-1 AGN}

For type-1 sources, the optical completeness factor, $\Theta\left(z, L_{15}\right)$, has been estimated using the mean value $\log \left(L_{15} / L_{R}\right)=0.23$ with a $1 \sigma$ dispersion of 0.25 (from the sample of Elvis et al. 1994). These values are in rough agreement with the derived value for the IRAS local sub-sample of type-1 AGN from Spinoglio et al. (1995; 0.05 with a $1 \sigma$ spread of 0.13 ). This ratio and its spread shows no significant dependence on $L_{15}$ over more than 4 decades of mid-IR luminosity and we assumed them to be constant with redshift.

In order to find the best fit solutions to the LF we have considered:

- two different behaviours for the faint slope of the LF: a) a fixed value $[\alpha=\alpha(0)]$ and, b) a dependence of $\alpha$ on $z$ of the form: $\alpha(z)=\alpha_{1} \exp \left(-\alpha_{2} * z\right)+\alpha_{3}$. A justification for this form is given in the following paragraphs;

- a fixed value for $z_{\text {cut }}$ equal to 2.0 in our parameterisation of the LF. Our relatively small number of sources, especially at high $z$, does not allow to sample correctly the existence of this redshift cutoff. We have therefore assumed a behaviour in the mid-IR similar to what is already known for the evolution of AGN in the optical (Boyle et al. 2000; Croom et al. 2004) and X-ray (Miyaji et al. 2000, 2001; La Franca et al. 2005; Hasinger et al. 2005), suggesting values for $z_{\text {cut }}$ in the range 1.7-2.5;

- a universe volume between $z=0$ and $z=4$, while the space density was integrated in the luminosity interval $\log L_{15}=$ $[42,47]$.

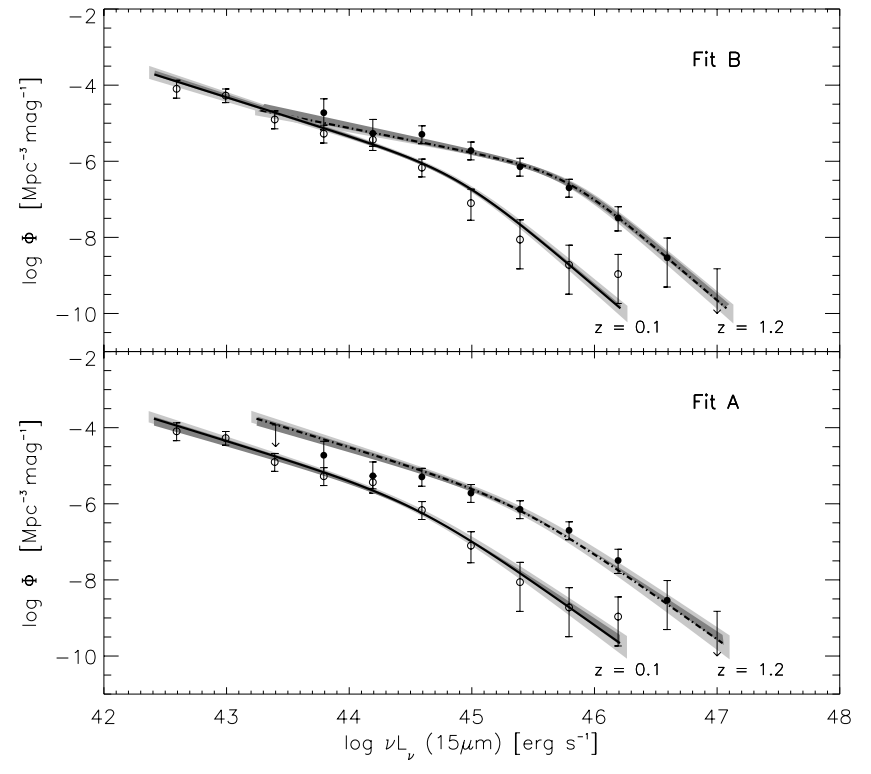

Fig. 4. Type-1 AGN local $(z=0.1$, continuous line) and high redshift $(z=1.2$, dash-dotted line) best fits to the $15 \mu \mathrm{m}$ Luminosity Function assuming a redshift dependent faint slope (Fit "B", top panel) and a PLE model (Fit "A", bottom panel). Open circles represent the observed space density of the local population (dominated mainly by RMS sources) while filled circles represent the observed space density of the high redshift population (dominated by ELAIS sources). Poissonian errors at $1 \sigma$ confidence level are shown. The dark-grey shaded areas (quite narrow and thus barely visible) represent the uncertainty introduced by the assumptions used to estimate the optical incompleteness of the samples, while the light-grey shaded areas correspond to the $1 \sigma$ errors of the best fit parameters of the LF (see Sect. 3.2).

In total, 72 (27 ELAIS + 41 RMS + 4 ISO-Deep) type-1 AGN were used to derive the LF. The best solutions found for each parameter $( \pm 1 \sigma$ confidence levels $)$ and the $2 \mathrm{D}-\mathrm{KS}$ probabilities are presented in Table 2 in the rows labeled " $A$ " and "B" for the fixed and variable faint slope models respectively.

In Fig. 4 we show the observed space density distribution and the best fit models in two redshift intervals: $z=[0,0.2]$, where the IRAS sources dominate, and $z=[0.2,2.2]$, mainly populated by ISO sources. The large interval at high redshifts $(z=[0.2,2.2])$ was chosen only for representation purposes, to assure a significant number of observed sources in each luminosity bin. In order to correct for evolution within the redshift intervals, the observed space density distribution is plotted following La Franca et al. (1997). The expected number of sources given by the model in each bin $\left(N^{\text {model }}\right)$ is computed and compared to the observed number of AGN in the bin $\left(N^{\text {data }}\right)$. The ratio $N^{\text {data }} / N^{\text {model }}$ for each bin is then multiplied for the value of the LF at the corresponding central luminosity and redshift value of the bin. The plotted error bars correspond to the $1 \sigma$ Poisson distribution and were estimated following Gehrels (1986). Space density upper limits are given where sources are expected by the model but not observed. The $1 \sigma$ dispersion for the LF was computed according to the $1 \sigma$ uncertainties of its parameters, and represented as a light-grey shaded area in Fig. 4. 
Table 2. Parameter values of the fit of the luminosity function.

\begin{tabular}{|c|c|c|c|c|c|c|c|c|c|c|}
\hline $\begin{array}{l}\text { Model } \\
\text { (I) }\end{array}$ & $\begin{array}{l}\alpha_{1} \\
\text { (II) }\end{array}$ & $\begin{array}{l}\alpha_{2} \\
\text { (III) }\end{array}$ & $\begin{array}{l}\alpha_{3} \\
\text { (IV) }\end{array}$ & $\begin{array}{l}\beta \\
\text { (V) }\end{array}$ & $\begin{array}{c}\log L_{15}^{*} a \\
(\mathrm{VI})\end{array}$ & $\begin{array}{c}k_{L} \\
(\mathrm{VII}) \\
\end{array}$ & $\begin{array}{l}z_{\text {cut }} \\
\text { (VIII) }\end{array}$ & $\begin{array}{c}\log \Phi^{* b} \\
\text { (IX) }\end{array}$ & $\begin{array}{c}\mathrm{CIRB}(\%)^{c} \\
(\mathrm{X})\end{array}$ & $\begin{array}{c}P_{2 \mathrm{DKS}} \\
(\mathrm{XI}) \\
\end{array}$ \\
\hline \multicolumn{11}{|c|}{ Type-1 AGN } \\
\hline A & 0.00 (fixed) & 0.00 (fixed) & $1.08_{-0.11}^{+0.09}$ & $2.29_{-0.16}^{+0.36}$ & $44.45_{-0.08}^{+0.08}$ & $2.78_{-0.18}^{+0.23}$ & 2.00 & $-5.78 \pm 0.03$ & $12.1_{-25}^{+7.7}\left(4.5_{-1.0}^{+2.9}\right)$ & 0.11 \\
\hline B & $0.69_{-0.08}^{+0.07}$ & $5.92_{-0.12}^{+0.17}$ & $0.64_{-0.11}^{+0.07}$ & $2.76_{-0.26}^{+0.21}$ & $44.78_{-0.06}^{+0.07}$ & $2.87_{-0.15}^{+0.27}$ & 2.00 & $-6.26 \pm 0.03$ & $4.2_{-1.0}^{+1.1}\left(1.6_{-0.4}^{+0.3}\right)$ & 0.35 \\
\hline \multicolumn{11}{|c|}{ Type-2 AGN (NGC 1068 SED) } \\
\hline $\mathrm{C}$ & 0.00 (fixed) & 0.00 (fixed) & $0.78_{-0.15}^{+0.10}$ & $2.67_{-0.35}^{+0.41}$ & $44.15_{-0.21}^{+0.20}$ & $1.79_{-0.57}^{+0.33}$ & $\ldots$ & $-4.77 \pm 0.03$ & $9.2_{-3.3}^{+6.1}\left(3.4_{-1.6}^{+1.2}\right)$ & 0.18 \\
\hline $\mathrm{D}$ & $0.90_{-0.14}^{+0.12}$ & $6.00^{* *}$ & $0.00^{-0.15}$ & $2.66_{-0.25}^{+0.35}$ & $44.11_{-0.16}^{-0.21}$ & $2.04_{-0.60}^{+0.70}$ & $\cdots$ & $-4.69 \pm 0.03$ & $5.5_{-1.5}^{+2.6}\left(2.0_{-0.8}^{+1.0}\right)$ & 0.33 \\
\hline \multicolumn{11}{|c|}{ Type-2 AGN (Circinus SED) } \\
\hline $\mathrm{E}$ & 0.00 (fixed) & 0.00 (fixed) & $0.76_{-0.14}^{+0.13}$ & $2.73_{-0.33}^{+0.42}$ & $44.15_{-020}^{+0.24}$ & $2.50_{-0.50}^{+0.55}$ & & $-4.75 \pm 0.03$ & $14.6_{-5}^{+8.4}\left(5.4_{-19}^{+3.1}\right)$ & 0.16 \\
\hline $\mathrm{F}$ & $0.87_{-0.14}^{+0.12}$ & $6.00^{* *}$ & $0.00^{-0.14}$ & $2.68_{-0.24}^{+0.26}$ & $44.10_{-0.12}^{+0.13}$ & $2.55_{-0.54}^{+0.52}$ & $\ldots$ & $-4.66 \pm 0.03$ & $7.7_{-2.1}^{+3.0}\left(2.9_{-0.8}^{+1.1}\right)$ & 0.49 \\
\hline
\end{tabular}

${ }^{a} L_{15}^{*}$ corresponds to $v L_{v}$ at $15 \mu \mathrm{m}$ and is given in $\mathrm{erg} \mathrm{s}^{-1} .{ }^{b}$ The normalisation, $\log \Phi^{*}$, is given in $\mathrm{Mpc}^{-3} \mathrm{mag}^{-1}$. ${ }^{c}$ Contribution $v I_{v}$ to the CIRB at $15 \mu \mathrm{m}$ in units of $10^{-11} \mathrm{~W} \mathrm{~m}^{-2} \mathrm{sr}^{-1}$. In parenthesis we report the $\%$ of the CIRB produced by the fit given a CIRB value of $2.7 \mathrm{nW} \mathrm{m}^{-2} \mathrm{sr}^{-1}$.

** The values of these parameters reached the physical limit imposed in our minimisation process. These constraints were imposed to the models in order to not produce unphysical solutions at high redshift due to the low statistics. Therefore, no errors are reported for these parameters.

Beside the natural statistical uncertainties due to the limited number of sources used in our analysis, our fitting technique is affected by possible errors due the assumed average value of the $L_{15} / L_{R}$ ratio used to correct for the spectroscopic incompleteness of the samples. In order to estimate these uncertainties we have recomputed the LF best fit solutions assuming two extreme cases for the $\log \left(L_{15} / L_{R}\right)$ ratio. In particular, we have used as the average value of $\log \left(L_{15} / L_{R}\right)$ our best estimate $(0.23)$ plus or minus the observed $1 \sigma$ dispersion $(0.25)$. Even under these extreme assumptions, the parameters of the LF result to be within the errors quoted in Table 2. The darkgrey shaded areas in both panels of Fig. 4 show the corresponding $1 \sigma$ range of uncertainty introduced in the estimate of the space density of type-1 AGN. As it is clearly seen in the figure, the statistical errors due to the limited number of sources used in our analysis dominate over the uncertainties due to the assumptions on the average value of the $\log \left(L_{15} / L_{R}\right)$ ratio. For example, the uncertainty introduced in the estimated density of type-1 AGN by the assumed $L_{15} / L_{R}$ relation is $\sim 10 \%$ at $L_{15} \sim L_{15}^{*}$, while the statistical uncertainty due to the limited number of sources is larger than $20 \%$. For these reasons, in order to compute the errors over the parameters of the LF and any derived quantity (e.g. integral counts and redshift distributions), we neglected the uncertainties related to the assumptions on the average value of the $\log \left(L_{15} / L_{R}\right)$ ratio.

We observe that although the overall fit by the PLE model (model A in Table 2, Fig. 4 bottom panel) is in reasonable agreement with the observed data, it overpredicts by a small amount $(\sim 1 \sigma)$, but systematically over 5 luminosity bins, the number of the low luminosity $\left(L_{15}(z)<L_{15}^{*}\right)$ and high redshift $(z=1.2)$ type-1 AGN. This is the main reason why a dependence of the faint slope on the redshift was included. The introduction of this dependence translates into a luminosity dependent luminosity evolution (LDLE) for the faint part of the LF (model B in Table 2). In this case, a better agreement between the model and the data is obtained (Fig. 4, top panel) as shown by the increment of the $2 \mathrm{D}-\mathrm{KS}$ probability (0.35 vs. 0.11$)$.
The measured rate of luminosity evolution $\left(k_{L}\right)$ is largely independent of the adopted relation between $\alpha$ and $z$. The best fit values, $k_{L} \sim 2.7-2.9$, are similar, within the errors, to those already found for these objects in the optical and X-ray wavelengths, where the evolution rate is between 2.5 and 3.5 (La Franca et al. 1997; Boyle et al. 2000; Miyaji et al. 2000 \& 2001; Croom et al. 2004; La Franca et al. 2005; Hasinger et al. 2005). Therefore, according to this analysis, no difference is found in the evolution of mid-IR selected type-1 AGN in comparison to the ones selected in the optical and X-rays.

The $15 \mu \mathrm{m}$ integral counts and the observed redshift distribution for type-1 AGN can now be compared with the predictions derived from the best fit models. The results of this comparison are presented in the upper right and left panels of Fig. 5. The bottom panels show the corresponding relative errors as derived from the $1 \sigma$ uncertainties on the best fit parameters of the LF.

The expected integral counts (Fig. 5, left) given by both models (dashed line for model "A" and continuous line for model "B") provide a good representation of the observed data (shaded areas) down to $\sim 2 \mathrm{mJy}$. At fluxes below $\sim 2 \mathrm{mJy}$ the variable slope model agrees better with the ELAIS data, while the PLE model is more representative of the fainter population in the HDF and CFRS fields. At the faintest fluxes $\left(S_{15}=0.1 \mathrm{mJy}\right)$ the variable slope model underpredicts the observed counts by a factor $\sim 2$. The uncertainties on the predicted counts at this flux ( 20\%) are not large enough to justify the observed discrepancy.

We also find an overall good agreement between the redshift distribution of the observed data and the model predictions (Fig. 5, right). The largest discrepancy between the models occurs at the lowest redshift bin $(z=[0,0.5])$ where the fixed faint slope model (thin lines) overpredicts the number of sources observed in the ISOCAM fields by a factor $\sim 3$ (4 observed vs. $\sim 12$ expected), while the variable faint slope model provides a better representation of the observed distribution.

In summary, although the variable faint slope model provides a better overall fit to the LF and reproduces better 

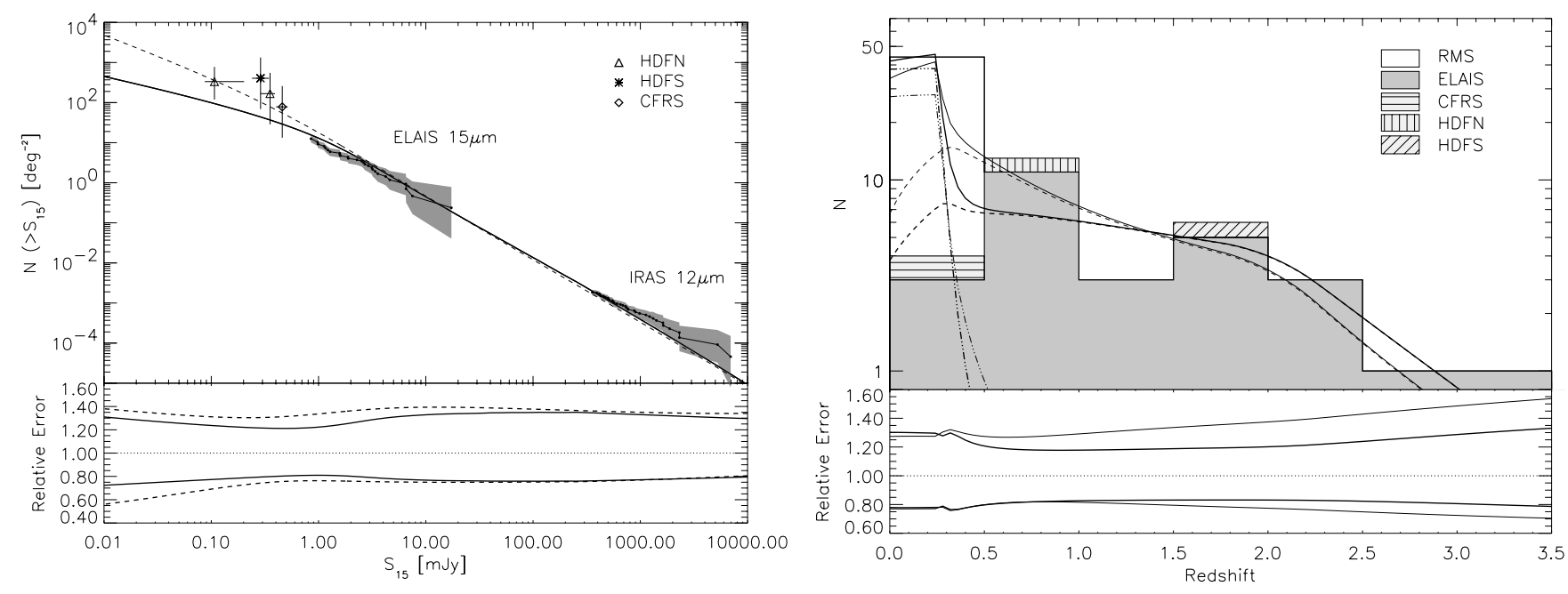

Fig. 5. Left: mid-IR integral counts for type-1 AGN. Shaded areas show the RMS and ELAIS observed counts, while symbols (triangle, star and diamonds) represent the ISO-Deep surveys. The thick solid line gives the predicted counts from the variable slope fit model ("B"). Expectations from the fixed faint slope model ("A") are plotted as a dashed line. All errors in the observed distributions are quoted at the $1 \sigma$ level and given by Poisson statistics. The bottom panel show the $1 \sigma$ relative error on the predicted counts due to the uncertainties on the best fit parameters of the LF (see text). Line types have the same meaning as in the above panel. Right: redshift distribution for type-1 AGN. The histogram represents the total number of observed sources in each redshift interval. Shaded regions outline the ISOCAM contribution, while the open histogram accounts for the contribution from IRAS sources. The lines are the predictions from the best fit model ("B"): thick dash-dotted for IRAS, thick dashed for ISO population, while the continuous thick line gives their sum. The expected distributions for the "A" model are plotted as above but using thin lines. The bottom panel shows the relative errors of the predictions for the entire mid-IR population (IRAS + ISO) only.

the $z$-distribution, it underestimates the faint mid-IR counts $\left(S_{15}<0.5 \mathrm{mJy}\right.$ ) by a factor of $\sim 4$. As both models ("A" fixed and "B" variable faint slope) are statistically acceptable, we conclude that given the presently available statistics the two models are equivalent within the errors.

M02 measured for the first time the evolution of type-1 AGN based on a preliminary mid-IR catalogue from the ELAIS-S1 field. The values found here for the fixed faint slope LF (model "A") are in agreement with what already found by M02 within $\sim 1 \sigma$ errors. The only exception is for the bright slope $(\beta)$, where the here derived value is flatter $\left(2.29_{-0.16}^{+0.36} \mathrm{vs}\right.$. $\left.2.89_{-0.26}^{+0.29}\right)$. This difference can be understood if we take into account the large uncertainties in the brighter luminosity intervals (in both M02 and our sample) due to the small number of sources. While the $2 \mathrm{D}-\mathrm{KS}$ test gives a $11 \%$ probability that the our sample of AGN is well represented by a non-evolving faint slope (model "A"), this probability decreases to $5 \%$ if we use the parameters values reported by M02 (Table 1 in M02).

\subsection{The evolution of type-2 AGN}

As for type-1 AGN, a relation between the intrinsic $15 \mu \mathrm{m}$ luminosity $\left(L_{15}\right)$ and the optical luminosity $\left(L_{R}\right)$ for type-2 AGN is required to correct for the optical incompleteness. FLF04 has shown that such a relation exists and found it to be valid for the whole mid-IR population (excluding type-1 sources) over more than 4 decades of mid-IR luminosity. In order to derive this relationship, optical identifications from RMS, ELAIS-S fields, HDF North (Aussel et al. 1999a,b) and South (Mann et al. 2002; Franceschini et al. 2003) were used. As opposed to type-1 sources, the ratio $\left(L_{15} / L_{R}\right)$ has a dependence on $L_{15}$ which can be expressed in the linear form

$\log \left(L_{15} / L_{R}\right)=0.47 \log L_{15}-5.02$,

where luminosities are expressed in solar units (see FLF04, Fig. 13). The data show an intrinsic $1 \sigma$ dispersion of 0.32 . This equation implies that $L_{15}$ increases $\sim 3$ times faster than $L_{R}$. The relation works for both nearby and distant objects (from $z=0$ up to $z=0.7$ ) and, therefore, has been used to estimate the fraction of sources lost due to the spectroscopic limit of the different surveys, i.e. the $\Theta\left(z, L_{15}\right)$ term used in the minimisation of the $S$ function (see Eq. (3)).

The LF was computed in the luminosity range $\log L_{15}=$ $[42,47]$ and in the redshift interval $z=[0,0.7]$. A total of 75 sources ( 25 from ELAIS and 50 from RMS) were used to derive the LF shape and evolution. Best fit values for the LF parameters, as well as the corresponding $2 \mathrm{D}-\mathrm{KS}$ probabilities, can be found in Table 2 for each of the two representative midIR $k$-corrections assumed.

For type-2 AGN, we followed the same fitting sequence as for type-1 AGN. The results for a fixed faint slope (PLE model) are found on Table 2 on rows labeled "C" (for the NGC 1068 $k$-correction) and " $\mathrm{E}$ " (for the Circinus $k$-correction). In both cases, the value of the $2 \mathrm{D}-\mathrm{KS}$ test $\left(P_{2 \mathrm{DKS}}=0.16\right.$ and 0.18 respectively) does not reject the PLE model, but an important source overprediction is again present in the model if compared to the observed data for the low luminosity and moderate- $z$ $(z=[0.1,0.6])$ part of the sample. A better fit is found by assuming an evolving faint slope of the LF parameterised as specified in Sect. 3.2. In this case, in order to avoid unphysical solutions due to the low statistics at high redshift, the $\alpha_{2}$ parameter was allowed to vary in the range $[0,6]$, while the $\alpha_{3}$ parameter 

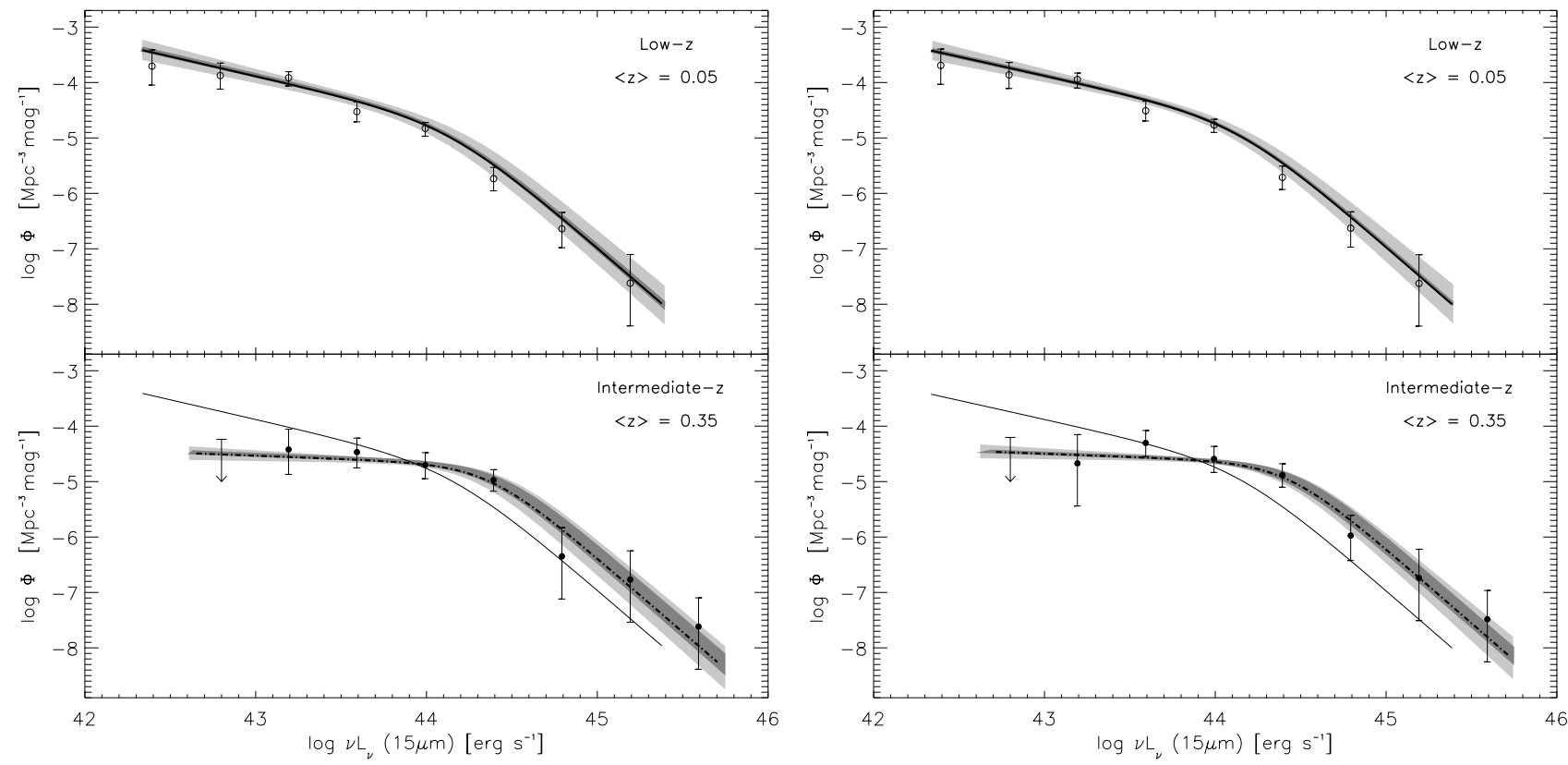

Fig. 6. Observed and best fit LF for type-2 AGN at $\langle z\rangle=0.05$ (continuous line) and $\langle z\rangle=0.35$ (dash-dotted line). The solutions with a redshift dependent faint slope, assuming a NGC 1068 ("D"; left panel) and Circinus ("F"; right panel) $k$-corrections, are shown. Symbols and shaded areas as in Fig. 4. Due to the small redshift intervals used, and for the sake of clarity, the plots have been divided into two panels for the low-redshift (top) and intermediate- $z$ sources (bottom). At intermediate- $z$, the thin line shows the low- $z$ best fit.

was allowed to vary in the range [0, 2]. Indeed, both parameters found a solution at the edges of the allowed ranges. The results can be found in Table 2 and Fig. 6, fits labeled " $D$ " and "F" for the NGC 1068 and Circinus $k$-corrections respectively.

Figure 6 shows the observed and predicted space density distribution of the type- $2 \mathrm{AGN}$ in two representative redshift intervals, low $-z(z=[0,0.1])$ where the IRAS sources dominate and intermediate- $z(z=[0.1,0.6])$, mainly populated by ISO sources. The overall best solution found for the two representative SED, NGC 1068 (left panel, model "D") and Circinus (right panel, model "F"), are plotted. The observed space density, errors, upper limits and the $1 \sigma$ confidence regions for the LF have been computed and plotted as described in Sect. 3.2. As observed for type-1 AGN, the errors introduced by the low statistics (light shaded area) are larger than the ones due to the assumptions on the $L_{15} / L_{R}$ relation (dark shaded area). For example, in this case, while the error introduced by the assumed $L_{15} / L_{R}$ relation in the determination of the density of type-2 AGN is $\sim 20 \%$ at the break luminosity $\left(L_{15}^{*}\right)$ of the LF, the limited statistics introduce, instead, a $\sim 60 \%$ uncertainty. Therefore, also for type-2 AGN, in order to compute the errors on the LF parameters and on any derived quantity (counts and redshift distributions) the uncertainties introduced by the assumptions on the value of the $L_{15} / L_{R}$ relation have been neglected.

The rate of evolution $k_{L}$ found for type- 2 AGN depends strongly on the assumed SED. If an NGC $1068 k$-correction is adopted, the rate of evolution is $k_{L} \sim 2.0 \pm 0.5$, lower than the one measured for type- 1 sources $\left(k_{L} \sim 2.8 \pm 0.3\right)$. On the other hand, an evolution rate $k_{L} \sim 2.5 \pm 0.5$ is obtained if the Circinus SED is assumed, closer to the one measured for type-1 AGN. These differences in $k_{L}$ are caused by the stronger $k$-correction introduced by the Circinus SED (Fig. 1), which generates higher intrinsic luminosities in the redshift range $[0.1,0.6]$ compared to those given by the NGC 1068 $k$-correction. In the other hand, the impact of the assumed SED on all the other fitted parameters is small (see Table 2 and Fig. 6).

For each best fit LF of the type-2 AGN, the predicted counts and redshift distributions were derived and compared with the observed data. Predictions at $15 \mu \mathrm{m}$ for the integral counts are given in the left panel of Fig. 7, while the derived redshift distribution is shown in the right panel of the same figure. In both panels of Fig. 7 a thick-dashed line represents the Circinus fixed slope model ("E"), a thick-continuous line the Circinus variable slope model ("F"), a thin-dashed line the NGC 1068 fixed slope model ("C") and a thick-continuous line the NGC 1068 variable slope model ("D”).

A good agreement, within the errors, between the models and the data is observed. At variance with the solutions found for the type-1 AGN (Sect. 3.2), although a higher 2DKS probability is obtained using a variable faint slope model, no major differences are observed in the integral counts down the faintest ELAIS flux $\left(\sim 1 \mathrm{mJy} ; \log S_{15} \sim 0\right)$. The differences between the various models only become significant at fainter fluxes $\left(S_{15} \leq 1 \mathrm{mJy}\right)$, especially at the fluxes reached by the Spitzer space observatory $\left(S_{24} \sim 0.01 \mathrm{mJy}\right.$; see Sect. 6$)$. Indeed, the relative errors are rather uniform, with mean values around 30-50\%. Therefore, the higher observed density of the deep ISO data (a factor $\sim 10$ ) is statistical significant. However, as previously mentioned (Sect. 2), the higher density of AGN found in these fields is partly due to the classification method used, which is not only based on a pure optical spectral classification. The predicted redshift distributions agree, within 

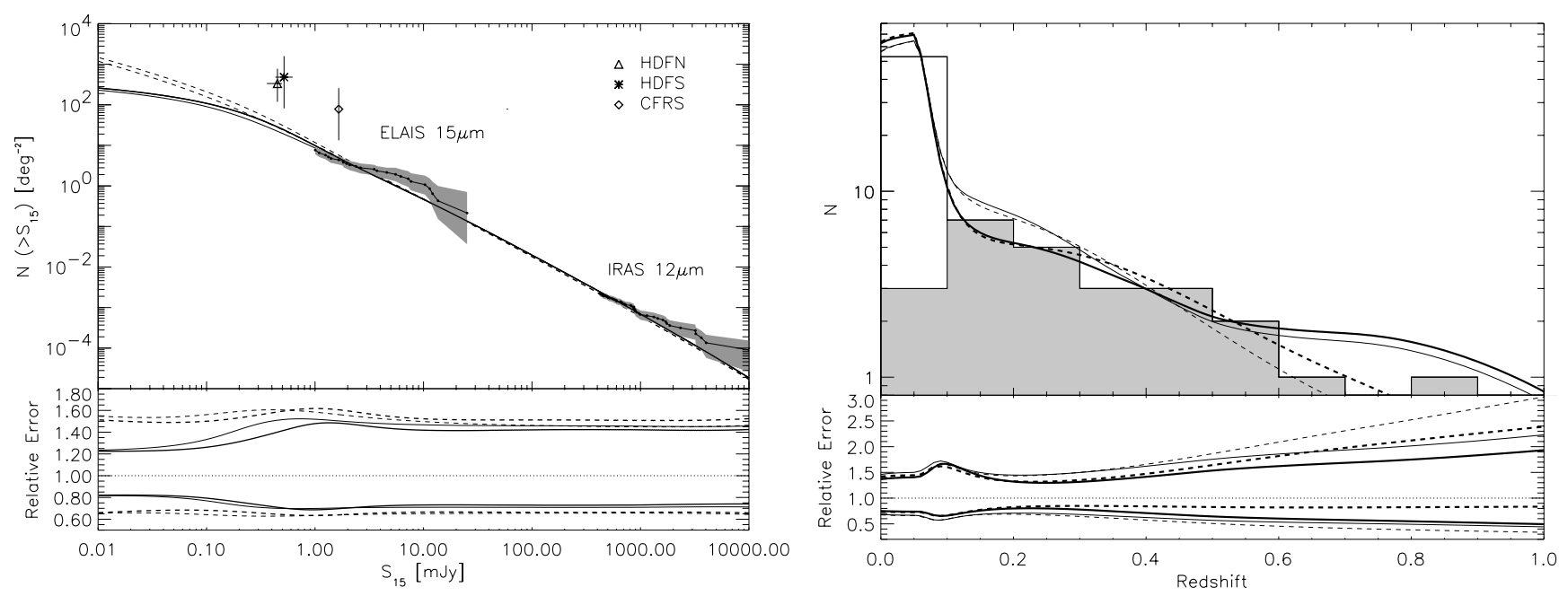

Fig. 7. Left: observed and predicted integral counts for type- 2 sources using the the two representative SED: NGC 1068 (thin dashed for the "C" model and thin continuous for the "D" model) and Circinus (thick dashed for the "E" model and thick continuous for the "F" model). Right: observed and predicted type-2 AGN redshift distribution for the different models mentioned before. Lines as in left panel. Symbols and shaded area in both panels as in Fig. 5. Errors reported in the lower section of both panels have been computed and plotted as described in Fig. 5.

the errors, in the redshift interval where the LF was computed $(z=[0,0.7])$. The different behaviour observed at $z>0.7$ is understood by the different $k$-corrections obtained from the adopted SED. The estimated errors range from a factor $\sim 1.3$ at low redshift to $\sim 2.0$ at the limiting redshift used to fit the LF, and increase drastically when the predictions are extrapolated at higher redshifts.

\subsection{The missing fraction of $A G N$}

In the previous sections we have seen that a flattening with redshift of the faint slope of both type-1 and type- 2 luminosity functions is slightly favoured by the data. This flattening would disappear if $\sim 7$ additional type-1 AGN, in the luminosity-redshift range $\log L_{15}=[42,45]$ and $z=[0.2,3.2]$, and $\sim 6$ additional type- 2 AGN within $\log L_{15}=[42,44]$ and $z=[0.1,0.7]$ were found. We briefly discuss now two possible origins of incompleteness for the mid-IR AGN sample and their effects on the observed LF: i) objects not identified due to the spectro-photometric limit of completeness of the survey and; ii) possible misclassification of the objects.

The spectroscopic incompleteness at faint optical magnitudes of the ELAIS sample is represented by the factor $\Theta\left(z, L_{15}\right)$ (Sect. 3.1). The corrections introduced through this term are represented in Fig. 8 as a function of redshift and mid-IR luminosity. The shaded areas in the figure outline the region where type-1 (top) and type- 2 sources (bottom), fainter than the spectro-photometric limit of the surveys, are expected to be found. A darker area in the plot indicates that a higher fraction of correction has been applied (i.e. higher number of lost sources) and it spans from $\sim 1 \%$ (light-grey area) to $\sim 30 \%$ (darkest area) of the total sky area available in a given interval of redshift and luminosity. The correction computed corresponds to $\sim 4$ type- 1 and $\sim 1$ type- 2 sources in the $L_{15}-z$ interval used in the minimisation process. If the suggested flattening is really due to an incompleteness effect, then the possibly missed objects are expected to follow an optical-mid-IR relation different from the one measured for the identified fraction of the high redshift $I S O$ sources and in the local universe by IRAS $(z<0.1)$ and adopted in our estimate of $\Theta\left(z, L_{15}\right)$. In the case of type-1 AGN, if the average value of the assumed $\log \left(L_{15} / L_{R}\right)$ relation is increased by 2.5 times the observed dispersion of the relation (i.e. $\sim 0.86$ instead of 0.23 ), $\sim 7$ additional sources would be expected at low luminosity and high redshifts, thus avoiding the need for a flattening of the LF. This higher $L_{15} / L_{R}$ value could correspond, for example, to sources with a significantly larger amount of gas and dust with respect to the local samples of Elvis et al. (1994) and Spinoglio et al. (1995). For type-2 AGN, the relation derived by La Franca et al. (2004) implies that for the low luminosity $\left(L_{15}<L_{15}^{*}\right)$ and moderate redshift $(z \sim 0.1-0.5)$ sources, all the possible optical counterparts should have been observed. Only the high luminosity $\left(L_{15}>L_{15}^{*}\right)$, higher redshift $(z>0.5)$ sources are affected by incompleteness. Also in this case a significant higher value of the $L_{15} / L_{R}$ relation would be required in order to avoid the need for a flattening of the LF.

A second source of incompleteness could be spectroscopic mis-classification of the sources. The increase in the mean redshift of the ISO population with respect to the local IRAS sample (see Table 1) implies that a larger fraction of the host galaxy light is collected for a given size of the slit used for the optical spectroscopic identifications. The consequence is a dilution of AGN signatures that affects the measured equivalent widths (EW) and line ratios (see e.g. Moran et al. 2002). The effect is less significant for type- 1 sources due to the presence of easily identified broad emission lines caused by a direct view of the central energy source with a low level of obscuration. The situation is more complicated for type-2 AGN since their higher degree of obscuration suppresses an important fraction of the optical light from the nuclei. The host galaxy would then naturally contribute with a larger fraction to the observed optical light from the nuclear source, sometimes even dominating it. The effect is maximised for the intrinsically faint AGN, whose optical features will be highly diluted into the host galaxy 

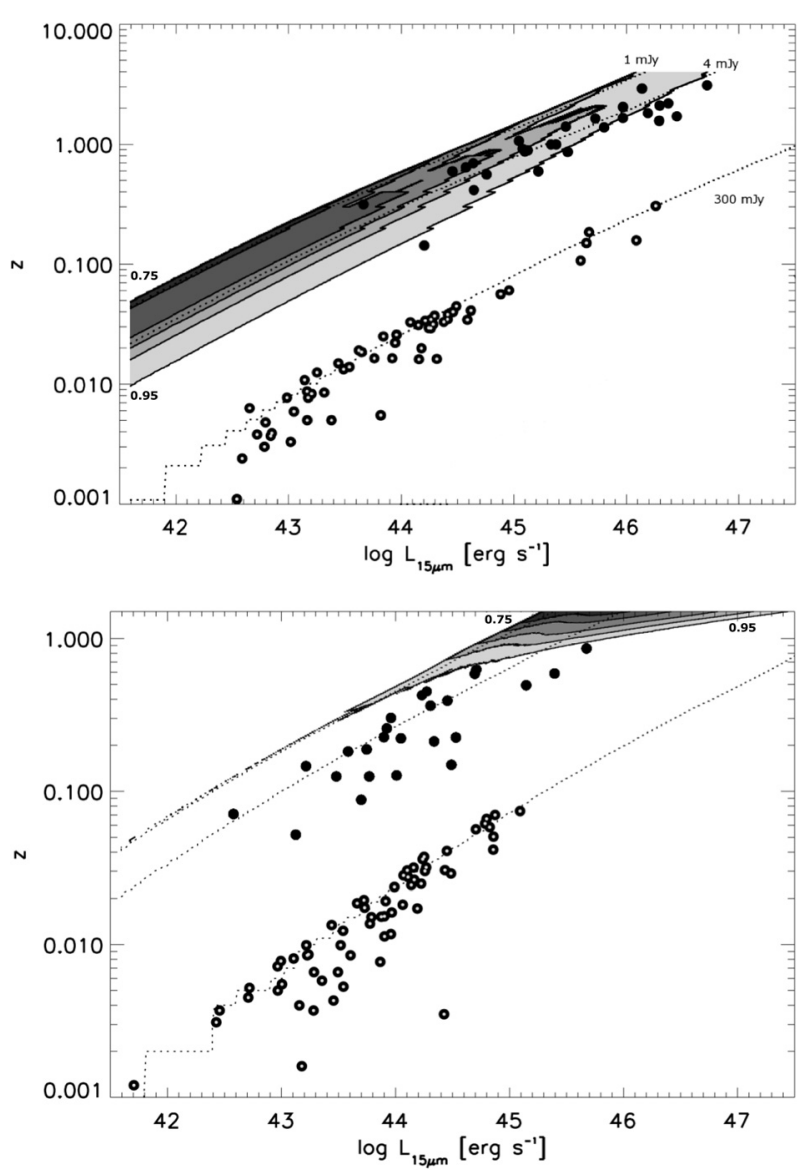

Fig. 8. Luminosity-redshift distribution of the type-1 (top) and type-2 (bottom) AGN. Shaded areas represents the fraction of incompleteness due to the optical spectroscopic limits. Contours range from $75 \%$ (black) to 95\% (light-gray) of the total area lost and have a 5\% step between them. For type-1 AGN $\sim 4$ missed sources are expected within $z=[0.1,1.2]$. Most missed type-2 AGN are only expected with $\log L_{15}>44.5$ at $z>0.7$, a redshift range outside the current analysis (see text). Only $\sim 1$ missed type- 2 source is expected to lie within $z=[0.3,0.5]$ and $L_{15}=[43.5,45.0]$. Dotted lines in both panels show three representative flux limits at 1,4 and $300 \mathrm{mJy}$.

spectrum. This effect could be relevant in producing the observed flattening at high redshift of the luminosity function.

Observations with XMM and Chandra have uncovered a not negligible number of AGN for which the classification given by their optical spectra does not correspond to the X-ray classification of the source (e.g. Fiore et al. 2000, 2003; Barger et al. 2005). In their analysis of deep ( 70 Ks long) XMM observations on ELAIS S1-5, La Franca et al. (in preparation) find that the fraction of misclassified type-1 AGN appears to be negligible. Vice-versa, in the case of type-2 AGN, the observations with XMM have revealed that about $10-20 \%$ of the ISO counterparts optically classified as no-AGN (i.e. normal and starburst galaxies) show X-ray luminosity consistent with AGN activity. This fraction, in the case of ELAIS-S, would imply that as many as 10 to 25 sources, classified in the optical as starburst or normal galaxies, may harbour an AGN that can contribute to the observed mid-IR flux. If this is the case, the number of true type-2 AGN may be higher by a factor $\sim 1.4-2.0$
Table 3. Parameter values of the fit of the mid-IR Luminosity Functions.

\begin{tabular}{lccccccccc}
\hline \hline & \multicolumn{3}{c}{ Type-1 } & \multicolumn{3}{c}{ Type-2 } & \multicolumn{3}{c}{ All } \\
& $\alpha$ & $\beta$ & $\log L_{15}^{*}$ & $\alpha$ & $\beta$ & $\log L_{15}^{*}$ & $\alpha$ & $\beta$ & $\log L_{15}^{*}$ \\
\hline RMS $^{a}$ & 0.0 & 2.1 & 42.8 & 0.0 & 2.5 & 43.2 & 0.3 & 2.1 & 43.4 \\
Xu03 $^{a, b}$ & - & - & - & - & - & - & 0.3 & 1.7 & 43.4 \\
${\text { Here(low- } z)^{c}}^{c}$ & 1.3 & 2.8 & 44.8 & 0.9 & 2.7 & 44.1 & - & - & - \\
Here(int- $z)^{c, d}$ & 0.7 & 2.8 & 45.4 & 0.0 & 2.7 & 44.6 & - & - & - \\
\hline
\end{tabular}

${ }^{a}$ Luminosities have been converted to $15 \mu \mathrm{m}$ using the adopted SEDs.

${ }^{b}$ From Xu et al. (2003).

${ }^{c}$ Best fit solutions for type-1 (model "B") and type-2 (Circinus SED, model "F").

${ }^{d}$ Intermediate redshift, $z=0.6$.

than that used in this analysis and this would have important consequences for the observed LF and its evolution.

We conclude that the observed flattening with redshift of the LF for low luminosity AGN can be explained if we assume that the incompleteness in the ELAIS sample was not properly modeled, for two possible reasons: (i) the unidentified fraction of sources may have a $L_{15} / L_{R}$ ratio different from that of the identified AGN or, (ii) more likely, a relevant fraction of type-2 AGN could have been mis-classified due to dilution of the optical nuclear spectra by the hosting galaxy. These sources of incompleteness are probably almost negligible for type-1 AGN (especially the last one), while our measure of the density of type-2 AGN has instead to be considered a lower limit.

\section{Comparison with previous works}

\subsection{The IRAS $12 \mu \mathrm{m}$ local luminosity function}

A local luminosity function (LLF) for type-1 and type-2 AGN was derived at $12 \mu \mathrm{m}$ by RMS. Their results can be directly compared to our findings at $z=0$. The values for the LF parameters reported by RMS and those computed by us are summarised in Table 3.

Two parameters of the LF computed by RMS, the faint slope $(\alpha)$ and the luminosity break $\left(L_{15}^{*}\right)$, differ significantly from our solutions for both the type-1 and the type-2 AGN. The difference in the value of the luminosity break cannot be explained by the SEDs conversion factors from 12 to $15 \mu \mathrm{m}$, since they imply a small change in the intrinsic luminosity of the sources $(\Delta \log L[12-15 \mu \mathrm{m}] \sim 0.0$ for type- 1 and $\sim 0.1$ for type-2 AGN). These discrepancies can instead be understood if we take into account that: (i) the faint slope was fixed a priori in the fitting procedure by RMS; (ii) the strong effect of evolution in the different luminosity bins was not considered by RMS and; (iii) our best fit is obtained by taking into account not only the local IRAS sources but also the high redshift ISOCAM population.

\subsection{The IRAS $25 \mu \mathrm{m}$ combined AGN sample}

A local combined (i.e. type-1 + type-2) AGN LF has been derived by Xu et al. (2003) at $25 \mu \mathrm{m}$. It is based on an IRAS colour selected $\left(S_{60 \mu \mathrm{m}} / S_{25 \mu \mathrm{m}}<5\right)$ sample obtained from a 


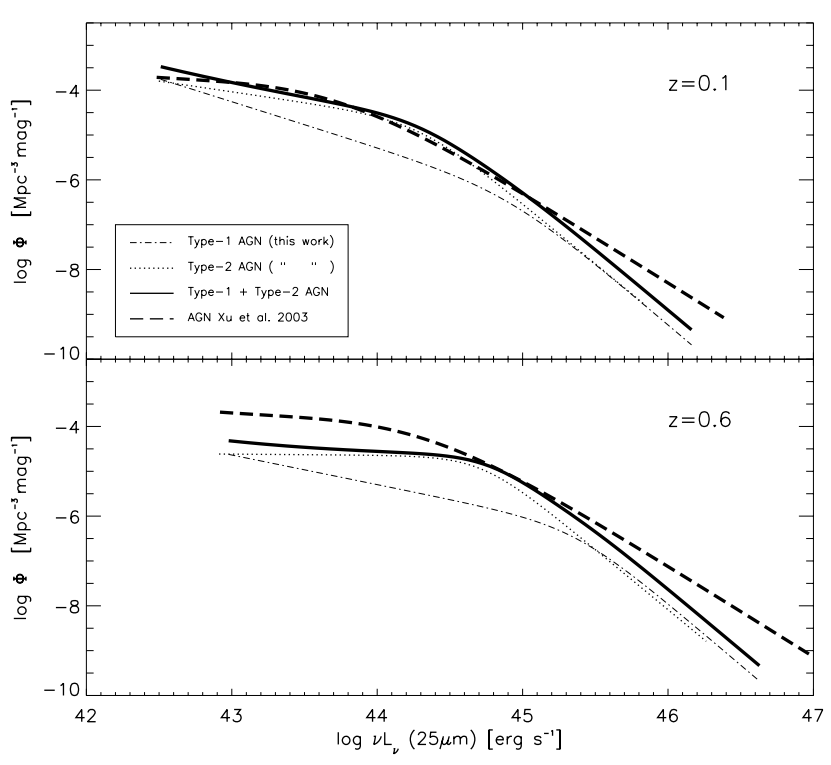

Fig. 9. Comparison of LFs at $25 \mu \mathrm{m}$. The derived $15 \mu \mathrm{m}$ LF for type- 1 (model "B") and type-2 (Circinus SED, model "F") AGN, converted to $25 \mu \mathrm{m}$ luminosities, are compared with the global AGN LF from $\mathrm{Xu}$ et al. (2003). The comparison is made in two different redshift intervals: $z=0.1$ (top panel) and $z=0.6$ (bottom panel). The values for the parameters of the LFs are reported in Table 3.

complete catalog compiled by Shupe et al. (1998). Assuming a luminosity evolution equal to the optical one (Boyle et al. 2000) and a set of SEDs with a dependence on luminosity, Xu et al. (2003) were able to derive the predictions for the AGN counts, the redshift distribution and the contribution to the cosmic background from the UV to the sub-mm wavelengths. A direct comparison of the two LFs can then be made by translating our $15 \mu \mathrm{m}$ luminosities into $25 \mu \mathrm{m}$ luminosities, using the adopted SEDs for each source class. The results of this comparison, in two different redshift intervals, can be found in Fig. 9 and Table 3 . We find a good agreement between the two LFs (dashed line for the $25 \mu \mathrm{m}$ selected sample; continuous line for our type-1 + type-2 $15 \mu \mathrm{m} \mathrm{LF})$ in the local universe $(z \sim 0.1)$. The difference found at intermediate- $z(z=0.6$, the maximum $z$ used to derive the type-2 LF) and low luminosities $L<L^{*}$ ) is mainly due to the observed decline in space density at this redshift for the obscured sources. The difference at higher luminosities $\left(L>L^{*}\right)$ can be explained by the stronger evolutionary rate found by Boyle et al. (2000) for optical selected type-1 $\mathrm{AGN}\left(k_{L} \sim 3.5\right)$ and used by Xu et al. (2003) in their analysis with respect to the values found in this work, $k \sim 2.0-2.9$ depending on the AGN type. The difference in the LF induced by these different $k_{L}$ parameters is already significant at $z \sim 0.6$.

\subsection{ISOCAM deep observations}

The deep observations carried out by the ISOCAM instrument on-board of ISO place interesting constraints on the shape and behaviour of the LF. For type-1 AGN they favour a PLE model scenario (dashed line in Fig. 5, left panel). This suggests that a small fraction of the low-luminosity ELAIS-S type-1 AGN may still be hidden within the rest of the mid-IR population. If this is the case, they do not follow the optical-mid-IR luminosity relation assumed for the rest of the type-1 sources as already discussed in Sect. 3.4.

The observations of type- 2 sources in these fields indicate that all our best fit solutions underestimate them by more than $1.5 \sigma$. This result is not surprising since most of the type-2 sources identified in these surveys have been classified using criteria different from the pure optical spectral classification (i.e. SED reconstruction, X-ray properties and radio emission). Indeed, this was the reason why we did not consider these sources in our minimisation process. A comparison between their observed space density and our predictions has to be made with care due to the different classification methods used.

Even if cosmic variance can increase the HDF-N, -S and CFRS quoted errors in Figs. 5 and 7 (by as much as 20-100\% in such a small fields, Somerville et al. 2004), it cannot fully justify the observed difference since in all these fields the observed counts are found to be systematically higher than our predictions.

\section{Contribution to the CIRB}

The contribution of AGN to the intensity of the Cosmic Background light in the Infrared (CIRB) has been derived for all the models presented in Table 2 . The intensity of the CIRB at $15 \mu \mathrm{m}$ for a given population has been computed as,

$I=\frac{1}{4 \pi} \int \mathrm{d} L_{15} \int \mathrm{d} z \frac{\mathrm{d} V}{\mathrm{~d} z} S_{v}\left(L_{15}, z\right) \Phi\left(L_{15}, z\right)$

where $S_{v}\left(L_{15}, z\right)$ represents the observed flux of a source with an intrinsic luminosity $L_{15}$ at redshift $z$. The integration has been performed for $\log L_{15}(z=0)=[42,47]$ up to $z=4$. For type- 2 sources the same redshift cutoff as for type- $1\left(z_{\text {cut }}=2.0\right)$ has been assumed. Column $\mathrm{X}$ in Table 2 summarises the expected background light for each model and the associated errors given by the $1 \sigma$ dispersion on the LF parameters as described in Sect. 3.2.

The integrated light from type- 1 AGN galaxies provides a contribution of $(4.2-12.1) \times 10^{-11} \mathrm{~W} \mathrm{~m}^{-2} \mathrm{sr}^{-1}$ (in units of $v I_{v}$ ) at $15 \mu \mathrm{m}$. This value corresponds to (1.6-4.5)\% of the $15 \mu \mathrm{m}$ background as reported by Elbaz et al. (2002) and confirmed by Metcalfe et al. $\left(2003,2.7 \times 10^{-9} \mathrm{~W} \mathrm{~m}^{-2} \mathrm{sr}^{-1}\right)$ using deep lensed observations with ISOCAM. Our predictions are in agreement with what previously estimated by M02 $(5.7 \times$ $\left.10^{-11} \mathrm{~W} \mathrm{~m}^{-2} \mathrm{sr}^{-1}\right)$.

The contribution of type-2 sources is found to be (5.5-14.6) $\times 10^{-11} \mathrm{~W} \mathrm{~m}^{-2} \mathrm{sr}^{-1}$, depending significantly on the assumed SED and the parameterisation for the evolution of the faint slope $[\alpha(z)]$, and accounts for $(2.0-5.4) \%$ of the CIRB at $15 \mu \mathrm{m}$. We note here that, even if the space density of the obscured sources, up to redshift 0.7 (where type-2 AGN are detected), is a factor 2-6 higher than that of the un-obscured ones (in the luminosity range $L_{15} \sim[43.5,45.0]$, around $L^{*}$, where most of the CIRB is produced), their contributions to the CIRB at $15 \mu \mathrm{m}$ are very similar. The main reason is that type-2 AGN have a strong $k$-correction imposed by the midIR SED. Moreover, the effect is increased by a slightly lower 


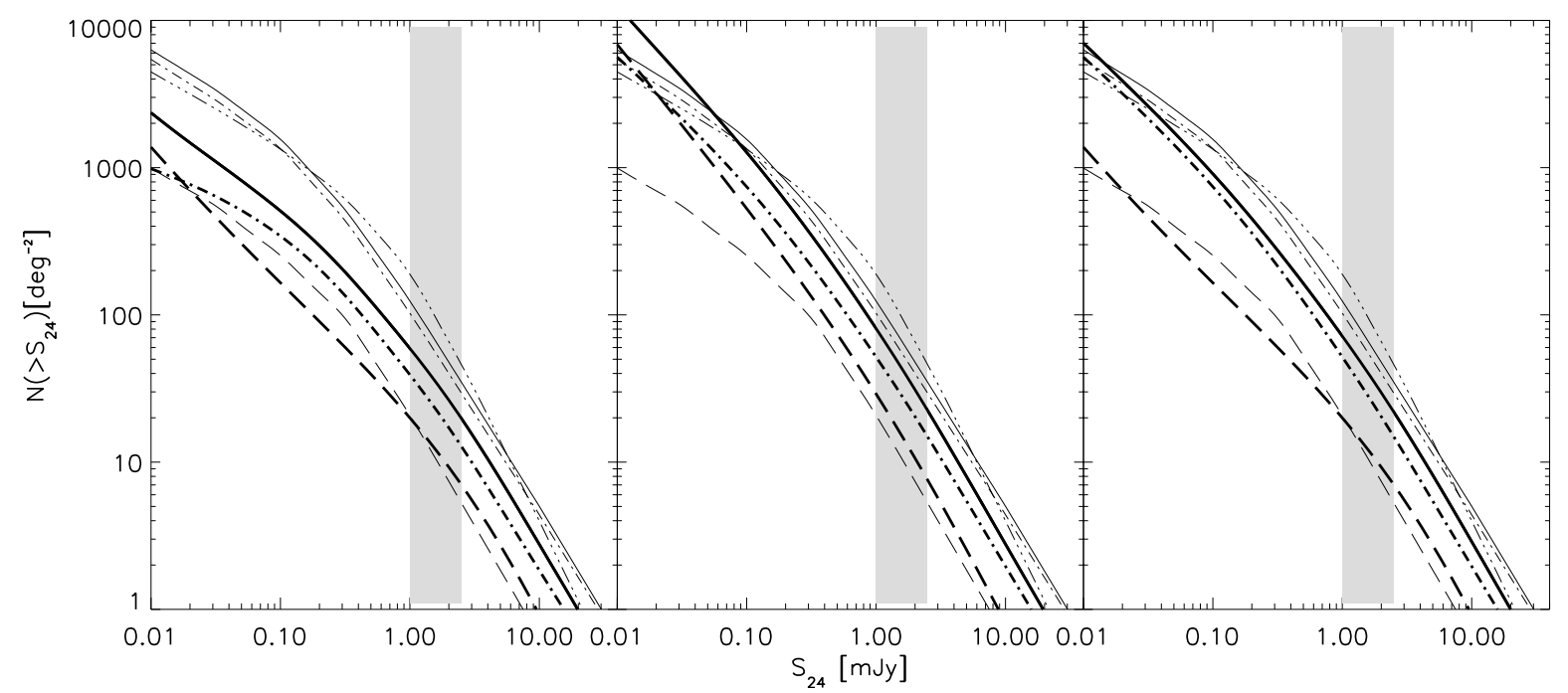

Fig. 10. The expected Spitzer MIPS integral counts for AGN at $24 \mu \mathrm{m}$ based on our best fit models (thick dashed for type-1, thick dot-dashed for type- 2 and thick continuous line for the total). The panels represent different combinations of type- 1 and type- 2 models from Table 2 . Left: models "B" and "F" for type-1 and type-2 respectively. Central: models "A" and "E". Right: models "B" and "E". For comparison, the expectations from the different AGN classes derived by Silva et al. (2004) are also shown (type-1: thin dashed line; type-2: thin dot-dashed; Total: thin continuous). The total AGN contribution by Xu et al. (2003) is represented by a thin triple-dot-dashed line. The light-grey shaded areas represent the expected flux range at $24 \mu \mathrm{m}$ of a source with a $15 \mu \mathrm{m}$ flux of $1 \mathrm{mJy}$ given the assumed type- 1 and type- 2 SED.

evolution rate than type-1 AGN, and a significant decline of the faint space density at higher $z$.

The total estimated contribution of AGN represents $\sim 4-10 \%$ of the total light observed in the mid-IR. This fraction is about half of the one ascribed to the AGN by M02. M02 study was limited to type-1 AGN, and assumptions based on the unified model and the local ratio of type- 2 to type-1 $(\sim 4$, Maiolino \& Rieke 1995) were made to derive their contribution. The smaller ratio of type- 2 to type- 1 found in our fields can explain the observed difference in our estimates.

Mid-IR studies based on IRAS and ISO observations have indirectly estimated a contribution of AGN to the CIRB not larger than 5-10\% (e.g. Malkan \& Stecker 1998; Franceschini et al. 2001; Xu et al. 2001, 2003). This is the maximum room "left" in their models by the strongly evolving starburst population. The total contribution was in any case uncertain since mid-IR selected type-2 AGN and starburst galaxies were treated as a single population. Another estimate of this contribution comes from the X-ray band $(0.5-10 \mathrm{keV})$, which offers a better wavelength regime where to select and identify obscured sources (unless the sources are Compton-thick, $N_{\mathrm{H}}>10^{25} \mathrm{~cm}^{-2}$ ). A cross-correlation of X-ray and IR sources detected by deep observations in the Chandra Deep field North (CDFN; Brandt et al. 2001) and in the Lockman Hole (Hasinger et al. 2001) allowed Fadda et al. (2002) to estimate the maximum fraction of the CIRB produced by AGN. This fraction is found to be of $(17 \pm 6) \%$, a factor $2-4$ higher than our predictions.

On the basis of the hard X-ray determination of the LF and semi-empirical SEDs (linking the X-ray to the Infrared), Silva et al. (2004) have derived the contribution of AGN and their host galaxy to the CIRB. For type-1 AGN, the agreement between their results $\left(\sim 7 \times 10^{-11} \mathrm{~W} \mathrm{~m}^{-2} \mathrm{sr}^{-1}\right)$ and the ones presented here is very good.
There is instead a significant difference for type-2 sources. The predictions from Silva et al. $\left(2004,3 \times 10^{-10} \mathrm{~W} \mathrm{~m}^{-2} \mathrm{sr}^{-1}\right)$ are, in the most favourable case (model "E"), at least a factor of 2 higher than ours and closer to the numbers provided by Fadda et al. (2002). The higher efficiency of the hard X-ray observations to select obscured AGN can explain the discrepancy between our results and the ones presented by Fadda et al. (2002) and Silva et al. (2004).

The conclusion drawn from the comparison with the above mentioned studies is that, as discussed in Sect. 3.4, it is likely that a significant fraction of type- 2 AGN is hidden within the rest of the mid-IR selected population, the normal and starburst galaxies.

\section{Mid-IR counts predictions for Spitzer}

The Spitzer space telescope is currently performing several deep mid- and far-infrared observations in selected areas of the sky. Following the results presented in this paper we have estimated the expected number of AGN sources as a function of the flux in the Spitzer mid-IR band. The derived integral counts for the MIPS instrument at $24 \mu \mathrm{m}$ are shown in Fig. 10. The results are given in three panels that correspond to three different combinations of type- 1 and type- 2 models (Table 2). Our estimates are compared to the total contribution of AGN from the models of Xu et al. (2003) and Silva et al. (2004). In the case of the Silva et al. (2004) model the individual contributions from type-1 and type-2 AGN are also shown. Best fit solutions with a redshift dependence on the faint slope are plotted in the left panel. The central panel presents the expectations assuming the best fits without any evolution for the faint slope of the LF. Finally, the combination of type- 1 and type- 2 models that better fits the expectations from Silva et al. (2004) is given in the right panel. 
At a $24 \mu \mathrm{m}$ flux of $0.01 \mathrm{mJy}$ the total AGN counts given by our best fit solutions underestimate the predictions by Silva et al. (2004) and Xu et al. (2003) by a factor of 2-3 (Fig. 10, left). With these models we predict to find $\sim 1200_{-300}^{+420}$ type- 1 and $\sim 1000_{-250}^{+350}$ type- 2 optically classified AGN per sq. degree down to a Spitzer flux limit of $S_{24 \mu \mathrm{m}}=0.01 \mathrm{mJy}$. If compared to the individual contributions of type- 1 and type- 2 AGN given by the Silva et al. (2004) model, we note that the large disagreement is mainly due to the low number of type-2 AGN expected in our models (a factor $\sim 5$ lower) and caused by the rapid flattening with $z$ of the faint LF slope. The large effect of the LF faint slope decline in the counts is evident if compared to the PLE models predictions plotted in the central panel. The PLE models overestimate the $24 \mu \mathrm{m}$ counts by both Xu et al. (2003) and Silva et al. (2004) below $0.06 \mathrm{mJy}$. A combination of the model "B" (variable LF faint slope for type-1 AGN) and model "E" (fixed faint slope model for type-2 AGN) produces the best approximation to the counts derived by the Silva et al. (2004) model based on the AGN hard X-ray LF (Fig. 10, right). If this is the case, then $\sim 1200$ type- 1 and $~ 5600$ type- 2 AGN persq. degree are expected brighter than $0.01 \mathrm{mJy}$ at $24 \mu \mathrm{m}$.

We want to note here that: i) although the expected Spitzer $/ 24 \mu \mathrm{m}$ integral counts derived by our different models differ by factors as large as $\sim 6-10$, they are very similar down to the flux limits of the ELAIS-South Surveys (shaded area in Fig. 10), responsible for the majority of the sources observed at high redshift in our sample; ii) even if some of our models produce counts comparable to the ones derived from X-ray LFs at $S_{24 \mu \mathrm{m}}=0.01 \mathrm{mJy}$ (Fig. 10, central and right panels), they are always lower at brighter fluxes. This second point explains the observed difference (by a factor of $\sim 3$ ) between our models and Silva et al. (2004) in the contribution of AGN to the CIRB.

\section{Conclusions}

Combining ISO $/ 15 \mu \mathrm{m}$ observations in ELAIS fields, HDF-N, HDF-S and the CFRS with the local IR population detected by IRAS at $12 \mu \mathrm{m}$, we have derived the evolution for the mid-IR selected and optically classified AGN. While a similar study had been done for QSOs+Seyfert-1 sources (Matute et al. 2002), we have presented here for the first time the rate of evolution shown by obscured, type-2 AGN. Our results are:

- Type-1 AGN evolve following a double power law LF and a rate of evolution of the form $(1+z)^{k_{L}}$ with $k_{L} \sim 2.9$. The data are consistent, within the errors, with a PLE model. However, a flattening at high redshift of the faint luminosity end of the luminosity function is marginally favoured by the data.

- Type-2 sources evolve with a slightly lower rate than type-1, with $k_{L}$ ranging from $\sim 1.8$ to $\sim 2.6$ depending strongly on the assumed SED used to compute the $k$-correction. The best fit solution favours a luminosity dependent luminosity evolution (LDLE) model, while the PLE model is statistically acceptable. However, integral counts reported for the deep $15 \mu \mathrm{m}$ observations in the HDF-N, -S and CFRS suggest an evolutionary scenario closer to the PLE model. We expect a significant number of type-2 AGN to be hidden within the optically classified normal and starburst galaxies.

- In the volume of the universe where both types of sources (type-1 and type-2) are observed $(z=[0,0.7])$ the ratio of type-2 to type-1 is $\sim 2-6$. This value is in agreement, within the errors, with the one measured from the local optically selected samples, where a ratio close to 4 is found (Maiolino \& Rieke 1995).

- The total contribution of AGN to the CIRB at $15 \mu \mathrm{m}$ is of the order of $\sim 4-10 \%$ of the total background light measured by Metcalfe et al. (2003), divided approximately equally between the two classes (type- 1 and type-2). The contribution of type-1 sources is in good agreement with the results presented in previous studies (Matute et al. 2002). Comparison with results from X-ray selected samples (e.g. Fadda et al. 2002; Silva et al. 2004) shows that a significant fraction of obscured type- 2 sources are missed in our sample. We argued that the optical spectral classification of the mid-IR sample, and not the mid-IR selection, is the principal cause of the missed fraction.

- Estimates of the number of mid-IR selected and optically classified AGN, expected from Spitzer at $24 \mu \mathrm{m}$, are given according to the best model results. We expect $\sim 1200$ type- 1 and $\sim 1000$ type- 2 optically classified AGN per sq. degree at a flux limit of $S_{24 \mu \mathrm{m}}=0.01 \mathrm{mJy}$. Our $24 \mu \mathrm{m}$ counts derived for type-1 sources agree very well with previous works. At a flux limit of $S_{24} \mu \mathrm{m}=0.01 \mathrm{mJy}$ the expected counts for the obscured population from our best fit models are a factor $\sim 5$ lower than the expectations from models based on X-ray LFs (e.g. Silva et al. 2004).

The results presented here for type-1 sources are quite robust since a high completeness is expected for these sources. Unlike type-1, type-2 AGN show a larger spread in their mid-IR and optical properties and a significant fraction can be misclassified as starburst or normal galaxies. As a consequence, the results for type-2 AGN derived here can only be considered as a lower limit to their true density and a first approximation to the evolution of these sources in the mid-IR. The true mid-IR space density of obscured sources has to be determined combining their mid-IR properties and the optical classification with the information available at other wavelengths, especially in X-rays.

Acknowledgements. The authors are grateful to the referee for helpful comments. This paper is based on observations collected at the European Southern Observatory, Chile (ESO No. 57.A0752, 58.B-0511, 59.B-0423, 61.B-0146, 62.P-0457, 67.A-0092(A), 68.A-0259(A), 69.A-0538(A) and 70.A-0362(A). This research has been partially supported by ASI, INAF and MIUR grants. I.M. acknowledges a Ph.D. grant from CNAA/INAF.

\section{References}

Alexander, D. M., Aussel, H., Bauer, F. E., et al. 2002, ApJ, 568, L85 Antonucci, R. 1993, ARA\&A, 31, 473

Aussel, H., Césarsky, C. J., Elbaz, D., \& Starck, J.L. 1999a, A\&A, 342,313

Aussel, H., Elbaz, D., Césarsky, C. J., \& Starck, J.L. 1999b, The Universe as seen by ISO, ed. P. Cox, \& M.F. Kessler (ESA Special Publication series), SP-427, 1023 
Balland, C., Devriendt, J. E. G., \& Silk, J. 2003, MNRAS, 343, 107

Barger, A. J., Cowie, L. L., Mushotzky, R. F., et al. 2005, AJ, 129, 578

Brandt, W. N., Alexander, D. M., Hornschemeier, A. E., et al. 2001, AJ, 122, 281

Boyle, B. J., Shanks, T., Croom, S. M., et al. 2000, MNRAS, 317, 1014

Clavel, J., Schulz, B., Barr, P., et al. 2000, A\&A, 357, 839

Comastri, A., Setti, G., Zamorani, G., \& Hasinger, G. 1995, A\&A, 296, 1

Croom, S. M., Smith, R. J., Boyle, B. J., et al. 2004, MNRAS, 349, 397

de Grijp, M. K. K., Miley, G. K., Lub, J., \& de Jong, T. 1985, Nature, 314,240

Di Matteo, T., Springel, V., \& Hernquist, L. 2005, Nature, 433, 604

Elbaz, D., Cesarsky, C. J., Chanial, P., et al. 2002, A\&A, 384, 848

Elvis, M., Wilkes, B. J., McDowell, J. C., et al. 1994, ApJS, 95, 1

Fadda, D., Flores, H., Hasinger, G., et al. 2002, A\&A, 383, 838

Fasano, G., \& Franceschini, A. 1987, MNRAS, 225, 155

Ferrarese, L., \& Merritt, D. 2000, ApJ, 539, L9

Fiore, F., La Franca, F., Vignali, C., et al. 2000, NewA, 5, 143

Fiore, F., Brusa, M., Cocchia, F., Baldi, A., Carangelo, N., et al. 2003, A\&A, 409, 79

Flores, H., Hammer, F., Thuan, T. X., et al. 1999, ApJ, 517, 148

Franceschini, A., Aussel, H., Cesarsky, C. J., Elbaz, D., \& Fadda, D. 2001, A\&A, 378, 1

Franceschini, A., Berta, S., Rigopoulou, D., et al. 2003, A\&A, 403, 501

Gehrels, N. 1986, ApJ, 303, 336

Gilli, R., Salvati, M., \& Hasinger, G. 2001, A\&A, 366, 407

Granato, G. L., Danese, L., \& Franceschini, A. 1997, ApJ, 486, 147

Granato, G. L., De Zotti, G., Silva, L., Bressan, A., \& Danese, L. 2004, ApJ, 600, 580

Gruppioni, C., Lari, C., Pozzi, F., et al. 2002, MNRAS, 335, 831

Hao, L., Strauss, M. A., Fan, X., et al. 2005, AJ, 129, 1795

Hasinger, G., Altieri, B., Arnaud, M., et al. 2001, A\&A, 365, L45

Hasinger, G., Miyaji, T., \& Schmidt, M. 2005, A\&A, 441, 417

La Franca, F., \& Cristiani, S. 1997, AJ, 113, 1517

La Franca, F., Fiore, F., Vignali, C., et al. 2002, ApJ, 570, 100

La Franca, F., Gruppioni, C., Matute, I., et al. 2004, AJ, 127, 3075 (FLF04)

La Franca, F., Fiore, F., Comastri, A., et al. 2005, ApJ, 635, 864

Lampton, M., Magon, B., \& Bowyer, S. 1976, ApJ, 207, 894

Lari, C., Pozzi, F., Gruppioni, C., et al. 2001, MNRAS, 325, 1173

Magorrian, J., Tremaine, S., Richstone, D., et al. 1998, AJ, 115, 2285

Maiolino, R., \& Rieke, G. H. 1995, ApJ, 454, 95

Malkan, M. A., \& Stecker, F. W. 1998, ApJ, 496, 13

Mann, R. G., Oliver, S., Carballo, R., et al. 2002, MNRAS, 332, 549

Marshall, H. L., Avni, Y., Tananbaum, H., \& Zamorani, G. 1983, ApJ, 269,35
Matute, I., La Franca, F., Pozzi, F., et al. 2002, MNRAS, 332, L11 (M02)

Menci, N., Fiore, F., Perola, G. C., \& Cavaliere, A. 2004, ApJ, 606, 58 Metcalfe, L., Kneib, J.-P., McBreen, B., et al. 2003, A\&A, 407, 791

Miley, G. K., Neugebauer, G., \& Soifer, B. T. 1985, ApJ, 293, 11

Miyaji, T., Hasinger, G., \& Schmidt, M. 2000, A\&A, 353, 25

Miyaji, T., Hasinger, G., \& Schmidt, M. 2001, A\&A, 369, 49

Moran, E. C., Filippenko, A. V., \& Chornock, R. 2002, ApJ, 579, L71

Moshir, M., et al. 1991, Explanatory Supplement to the IRAS Faint Source Survey, Version 2 (Pasadena: JPL)

Natali, F., Giallongo, E., Cristiani, S., \& La Franca, F. 1998, AJ, 115, 397

Nenkova, M., Ivezić, Ž., \& Elitzur, M. 2002, ApJ, 570, L9

Neugebauer, G., Soifer, B. T., \& Miley, G. K. 1986, ApJ, 308, 815

Oliva, E., Origlia, L., Maiolino, R., \& Moorwood, A. F. M. 1999, A\&A, 350, 9

Oliver, S., Rowan-Robinson, M., Alexander, D. M., et al. 2000, MNRAS, 316, 749

Oliver, S., Mann, R. G., Carballo, R., et al. 2002, MNRAS, 332, 536

Osterbrock, D. E. 1989, Astrophysics of Gaseous Nebulae and Active Galactic Nuclei (Mill Valley: Univ. Sci.)

Peacock, J. A. 1983, MNRAS, 217, 601

Pompilio, F., La Franca, F., \& Matt, G. 2000, A\&A, 353, 440

Pozzi, F., Ciliegi, P., Gruppioni, C., et al. 2003, MNRAS, 343, 1348

Pozzi, F., Gruppioni, C., Oliver, S., et al. 2004, ApJ, 609, 122

Rowan-Robinson, M., Lari, C., Perez-Fournon, I., et al. 2004, MNRAS, 351, 1290

Rush, B., Malkan, M. A., \& Spinoglio, L. 1993, ApJS, 89, 1 (RMS)

Sanders, D. B., Phinney, E. S., Neugebauer, G., Soifer, B. T., \& Matthews, K. 1989, ApJ, 347, 29

Shupe, D. L., Fan, F., Hacking, P. B., \& Huchra, J. P. 1998, ApJ, 501, 597

Silva, L., Maiolino, R., \& Granato, G. L. 2004, MNRAS, 355, 973

Somerville, R. S., Lee, K., Ferguson, H. C., et al. 2004, ApJ, 600, L171

Spinoglio, L., Malkan, M. A., Rush, B., et al. 1995, ApJ, 453, 616

Spoon, H. W. W., Keane, J. V., Tielens, A. G. G. M., et al. 2002, A\&A, 385,1022

Sturm, E., Lutz, D., Tran, D., et al. 2000, A\&A, 358, 481

Tremaine, S., Gebhardt, K., Bender, R., et al. 2002, ApJ, 574, 740

Tresse, L., Rola, C., Hammer, F., et al. 1996, MNRAS, 281, 847

Ueda, Y., Akiyama, M., Ohta, K., \& Miyaji, T. 2003, ApJ, 598, 886

Veilleux, S., \& Osterbrock, D. E. 1987, ApJS, 63, 295

Xu, C. K., Lonsdale, C. J., Shupe, D. L., O'linger, J., \& Masci, F. 2001, ApJ, 562, 179

Xu, C. K., Lonsdale, C. J., Shupe, D. L., et al. 2003, ApJ, 587, 90

York, D. G., et al. 2000, AJ, 126, 2125 